\title{
REPRESENTATIONS OF METAPLECTIC GROUPS II: HECKE ALGEBRA CORRESPONDENCES
}

\author{
WEE TECK GAN AND GORDAN SAVIN
}

\begin{abstract}
The metaplectic group is defined by its oscillator or Weil representation. Using the types of the Weil representations we define two Hecke algebras that govern two Bernstein's components containing the even and the odd Weil representation, respectively.
\end{abstract}

\section{INTRODUCTION}

Let $k$ be a $p$-adic field with $p$ odd. Let $V^{+}$and $V^{-}$be two quadratic spaces of dimension $2 n+1$, trivial discriminant, and trivial and non-trivial Hasse invariants, respectively. Then $\mathrm{SO}\left(V^{+}\right)$is a split, adjoint group of type $B_{n}$, while $\mathrm{SO}\left(V^{-}\right)$is its unique non-split inner form. Let $\mathcal{S}^{ \pm}$denote the category of smooth representations of $\mathrm{SO}\left(V^{ \pm}\right)$, and $\mathcal{S}_{0}^{ \pm}$the component (in the sense of Bernstein [Be] of $\mathcal{S}^{ \pm}$containing the trivial representation of $\mathrm{SO}\left(V^{ \pm}\right)$.

Let $W$ be a non-degenerate symplectic space of dimension $2 n$ over $k$. Let $\widetilde{\operatorname{Sp}}(W)$ be a non-trivial 2-fold central extension of $\operatorname{Sp}(W)$. Let $\mathcal{G}$ denote the category of genuine, smooth representations of $\widetilde{\operatorname{Sp}}(W)$. Let $\psi$ be a non-trivial additive character of $k$. Let $\omega_{\psi}$ be the Weil representation of $\widetilde{\mathrm{Sp}}(W)$, which depends on $\psi$ We. The Weil representation decomposes $\omega_{\psi}=\omega_{\psi}^{e} \oplus \omega_{\psi}^{o}$ into a sum of two irreducible representations, called even and odd Weil representations. Let $\mathcal{G}_{\psi}^{+}$and $\mathcal{G}_{\psi}^{-}$be the components of $\mathcal{G}$ containing $\omega_{\psi}^{e}$ and $\omega_{\psi}^{o}$, respectively.

The main result of this paper is an equivalence of categories $\mathcal{G}_{\psi}^{+}$and $\mathcal{S}_{0}^{+}$, and an equivalence of categories $\mathcal{G}_{\psi}^{-}$and $\mathcal{S}_{0}^{-}$. Under these equivalences, $\omega_{\psi}^{e}$ corresponds to the trivial representation of the split group $\mathrm{SO}\left(V^{+}\right)$while $\omega_{\psi}^{o}$ corresponds to the trivial representation of $\mathrm{SO}\left(V^{-}\right)$. More precisely, we describe two Hecke algebras, denoted by $H_{\psi}^{+}$and $H_{\psi}^{-}$, such that the subcategories $\mathcal{G}_{\psi}^{+}$and $\mathcal{G}_{\psi}^{-}$are equivalent to the categories of $\mathrm{H}_{\psi}^{+}$-modules and $\mathrm{H}_{\psi}^{-}$-modules, respectively. Via explicit isomorphisms, the algebras $H_{\psi}^{+}$and $H_{\psi}^{-}$are isomorphic to the affine Hecke algebras of $\mathrm{SO}\left(V^{+}\right)$and $\mathrm{SO}\left(V^{-}\right)$, respectively. While much of this approach is standard, a novelty in this paper is that we exploit minimal types of the Weil representation to define generators of $H_{\psi}^{+}$and $H_{\psi}^{-}$, and quickly prove relations satisfied by them.

The second result of this paper is a comparison of the correspondence of representations obtained in this paper with the theta correspondences. Recall that, by results of Kazhdan and Lusztig, the set of equivalence classes of irreducible

Received by the editors April 28, 2011 and, in revised form, May 9, 2012 and June 6, 2012.

2010 Mathematics Subject Classification. Primary 22E50; Secondary 11F27.

The first author was partially supported by NSF grant DMS0801071.

The second author was partially supported by DMS 0852429. 
representations in $\mathcal{S}_{0}^{+}$and $\mathcal{S}_{0}^{-}$can be partitioned into finite sets parameterized by conjugacy classes of pairs $(s, u)$,

$$
\operatorname{Irr}\left(\mathcal{S}_{0}^{+}\right) \cup \operatorname{Irr}\left(\mathcal{S}_{0}^{-}\right)=\bigcup \operatorname{Irr}(s, u),
$$

where $s$ is a semi-simple element in $\operatorname{Sp}_{2 n}(\mathbb{C})$ and $u$ is a unipotent element in $\operatorname{Sp}_{2 n}(\mathbb{C})$ such that sus $^{-1}=u^{q}$. Consequently, by the equivalences of categories obtained in this paper, there is a decomposition

$$
\operatorname{Irr}\left(\mathcal{G}_{\psi}^{+}\right) \cup \operatorname{Irr}\left(\mathcal{G}_{\psi}^{-}\right)=\bigcup \operatorname{Irr}_{\psi}(s, u) .
$$

We show that theta correspondences (that depend on $\psi$ ) for dual pairs $\operatorname{Sp}(W) \times$ $\mathrm{SO}\left(V^{ \pm}\right)$give a bijection of finite sets $\operatorname{Irr}(s, u)$ and $\operatorname{Irr}_{\psi}(s, u)$ for every pair $(s, u)$. In general this bijection is different from that given by the isomorphisms of Hecke algebras. A similar statement for dual pairs involving even orthogonal groups was proved by Aubert $\mathrm{Au}$.

\section{Classical groups}

Let $W$ be a non-degenerate symplectic space over $k$ of dimension $2 n$, and let $\langle\cdot, \cdot\rangle$ denote the symplectic form. Pick a basis of $W$ consisting of $\mathbf{e}_{i}$ and $\mathbf{e}_{i}^{*}, 1 \leq i \leq n$, such that $\left\langle\mathbf{e}_{i}, \mathbf{e}_{j}\right\rangle=\left\langle\mathbf{e}_{i}^{*}, \mathbf{e}_{j}^{*}\right\rangle=0$ and $\left\langle\mathbf{e}_{i}, \mathbf{e}_{j}^{*}\right\rangle=\delta_{i j}$. We have a decomposition $W=X+X^{*}$ where $X$ is spanned by $\mathbf{e}_{i}$ and $X^{*}$ is spanned by $\mathbf{e}_{i}^{*}$. The group $\operatorname{Sp}(W)$ has a maximal torus $T=\left(k^{*}\right)^{n}$ where $t=\left(t_{1}, \ldots, t_{n}\right) \in T$ acts on $\mathbf{e}_{i}$ and $\mathbf{e}_{i}^{*}$ by

$$
t\left(\mathbf{e}_{i}\right)=t_{i} \cdot \mathbf{e}_{i} \text { and } t\left(\mathbf{e}_{i}^{*}\right)=t_{i}^{-1} \cdot \mathbf{e}_{i}^{*} .
$$

Let $\Sigma=\left\{ \pm e_{i} \pm e_{j}\right\} \cup\left\{ \pm 2 e_{i} \mid 1 \leq i \leq n\right\}$ be the root system corresponding to $T$ where $\alpha=2 e_{i}$ is given by $\alpha(t)=t_{i}^{2}$. For every root $\alpha \in \Sigma$ there is a root subgroup $U_{\alpha} \cong k$ of $\operatorname{Sp}(W)$. For every $\alpha \in \Sigma$ we fix a map

$$
\Phi_{\alpha}: \mathrm{SL}_{2}(k) \rightarrow \mathrm{Sp}(W)
$$

such that $U_{\alpha}$ and $U_{-\alpha}$ are the images of the subgroups of unipotent upper and lower triangular matrices, respectively. We assume that $\Phi_{\alpha}$ and $\Phi_{-\alpha}$ are related by

$$
\Phi_{\alpha}\left(\begin{array}{ll}
a & b \\
c & d
\end{array}\right)=\Phi_{-\alpha}\left(\begin{array}{cc}
d & c \\
b & a
\end{array}\right) .
$$

If $\alpha=2 e_{i}$, we fix $\Phi_{\alpha}$ so that $\mathrm{SL}_{2}(k)$ acts, in the standard way, on the 2-dimensional subspace of $W$ spanned by $\mathbf{e}_{i}$ and $\mathbf{e}_{i}^{*}$, and fixes other basis vectors in $W$. If $\alpha=$ $e_{i}-e_{j}$, we fix $\Phi_{\alpha}$ so that $\mathrm{SL}_{2}(k)$ acts, in the standard way, on the 2-dimensional subspace of $X$ spanned by $\mathbf{e}_{i}$ and $\mathbf{e}_{j}$, and by the dual action on the 2-dimensional subspace of $X^{*}$ spanned by $\mathbf{e}_{i}^{*}$ and $\mathbf{e}_{j}^{*}$.

For any $l, 0 \leq l \leq n$, let $W_{l} \subseteq W$ be the span of $\mathbf{e}_{l+1}, \ldots \mathbf{e}_{n}$ and $\mathbf{e}_{l+1}^{*}, \ldots \mathbf{e}_{n}^{*}$. If $l=n$, we set $W_{n}=0$. Let $X_{l} \subseteq X$ be the span of $\mathbf{e}_{1}, \ldots, \mathbf{e}_{l}$. If $l=0$, we set $X_{0}=0$. Similarly, let $X_{l}^{*} \subseteq X^{*}$ be the span of $\mathbf{e}_{1}^{*}, \ldots, \mathbf{e}_{l}^{*}$. Then

$$
W=X_{l}+W_{l}+X_{l}^{*} \text {. }
$$

The stabilizer of the flag

$$
X_{1} \subseteq X_{2} \subseteq \ldots \subseteq X_{n}
$$

in $\operatorname{Sp}(W)$ is a Borel subgroup $B$ containing $T$. The corresponding set $\Delta$ of simple roots consists of $\alpha_{1}=e_{1}-e_{2}, \ldots, \alpha_{n-1}=e_{n-1}-e_{n}$ and $\alpha_{n}=2 e_{n}$. A parabolic subgroup $P$ is in a standard position if it contains $B$. All such parabolic subgroups 
are stabilizers of subflags of the flag $X_{1} \subseteq \ldots \subseteq X_{n}$. For example, the stabilizer of $X_{l}$ is a maximal parabolic $P$. We fix a Levi factor $M$ of $P$ so that it contains $T$. Equivalently, $M$ stabilizes $X_{l}^{*}$. Thus we have canonical isomorphisms

$$
M \cong \mathrm{GL}\left(X_{l}\right) \times \mathrm{Sp}\left(W_{l}\right) \cong \mathrm{GL}\left(X_{l}^{*}\right) \times \mathrm{Sp}\left(W_{l}\right) .
$$

Let $V$ be an orthogonal space over $k$ of dimension $2 n+1$ and discriminant 1 . Up to an isomorphism there are two such spaces: $V^{+}$and $V^{-}$, with trivial and nontrivial Hasse invariant, respectively. Let $(\cdot, \cdot)$ denote the corresponding symmetric bilinear form. Let $Y_{l} \subseteq V$ be a totally isotropic subspace of dimension $l$. Note that $l \leq n$ if $V \cong V^{+}$and $l \leq n-1$ if $V \cong V^{-}$. Then there exists another totally isotropic subspace $Y_{l}^{*}$ of dimension $l$ and an orthogonal subspace $V_{l}$ of discriminant 1 such that

$$
V \cong Y_{l}+V_{l}+Y_{l}^{*}
$$

Pick a basis $\mathbf{f}_{i}$ of $Y_{l}$ and a basis $\mathbf{f}_{i}^{*}$ of $Y_{l}^{*}, 1 \leq i \leq n$, such that $\left(\mathbf{f}_{i}, \mathbf{f}_{j}^{*}\right)=\delta_{i j}$. The group $\mathrm{SO}(V)$ contains a torus $T_{l}=\left(k^{*}\right)^{l}$ where $t=\left(t_{1}, \ldots, t_{l}\right) \in T_{l}$ acts trivially on $V_{l}$, and

$$
t\left(\mathbf{f}_{i}\right)=t_{i} \cdot \mathbf{f}_{i} \quad \text { and } \quad t\left(\mathbf{f}_{i}^{*}\right)=t_{i}^{-1} \cdot \mathbf{f}_{i}^{*} .
$$

Note that $T_{n}$ is a maximal torus of $\mathrm{SO}\left(V^{+}\right)$, while $T_{n-1}$ is a maximal split torus of $\mathrm{SO}\left(V^{-}\right)$. The stabilizer of $Y_{l}$ in $\mathrm{SO}(V)$ is a maximal parabolic $\operatorname{subgroup} Q$. We fix a Levi factor $L$ of $Q$ so that it stabilizes $Y_{l}^{*}$. Then

$$
L \cong \mathrm{GL}\left(Y_{l}\right) \times \mathrm{SO}\left(V_{l}\right) \cong \mathrm{GL}\left(Y_{l}^{*}\right) \times \mathrm{SO}\left(V_{l}\right) .
$$

\section{Open COMPACT SubGROUPS OF $\operatorname{Sp}(W)$}

Let $R$ be the ring of integers in $k$ and $\varpi$ a uniformizing element. Let $q$ be the order of the residue field $\mathfrak{f}=R / \varpi R$. Let $L$ be a lattice in $W$. Let $L^{*}$ be the dual lattice with respect to the symplectic form. A lattice $L$ is called good, if $\varpi L \subseteq L^{*} \subseteq L$. We fix a sequence of good lattices,

$$
L_{0} \subseteq L_{1} \subseteq \ldots \subseteq L_{n}
$$

where $L_{i}$ is the $R$-span of $\mathbf{e}_{j}$ for $1 \leq j \leq n$, $\frac{\mathbf{e}_{j}^{*}}{\varpi}$ for $j \leq i$, and $\mathbf{e}_{j}^{*}$ for $j>i$. Let $K_{i}$ be the stabilizer of the lattice $L_{i}$ in $\operatorname{Sp}(W)$. It is a maximal open compact subgroup, and contains a pro- $p$ subgroup $K_{i}^{+}$such that $K_{i} / K_{i}^{+} \cong \operatorname{Sp}_{2 i}(\mathfrak{f}) \times \operatorname{Sp}_{2(n-i)}(\mathfrak{f})$. The intersection of all $K_{i}$ is an Iwahori subgroup denoted by $I$. The double cosets with respect to $I$ can be described in terms of the affine Weyl group. If we view a root $\alpha$ as a linear functional on $\mathbb{E}=\mathbb{R} e_{1}+\cdots+\mathbb{R} e_{n}$ via the standard dot product then, for any $m \in \mathbb{Z}, \beta=\alpha+m$ is an affine functional on $\mathbb{E}$. Let $s_{\beta}$ be the reflection about the hyperplane in $\mathbb{E}$ where $\beta$ vanishes. The affine Weyl group $W_{a}$ is the group of affine transformations of $\mathbb{E}$ generated by these reflections. It is a semi-direct product of the Weyl group and a normal subgroup consisting of translations by vectors in $\mathbb{Z}^{n}$.

Let $\Delta_{a}=\Delta \cup\left\{\alpha_{0}\right\}$ be the set of simple affine roots where $\alpha_{0}=1-2 e_{1}$. Let $\mathcal{C} \subseteq \mathbb{E}$ consist of all $\left(x_{1}, \ldots, x_{n}\right)$ such that

$$
\frac{1}{2}>x_{1}>\ldots>x_{n}>0 .
$$


Then $\mathcal{C}$ is the chamber corresponding to $\Delta_{a}$ : its elements are all points in $\mathbb{E}$ where all simple roots take positive value.

As an abstract group, $W_{a}$ is generated by reflections $s_{0}, \ldots, s_{n}$ corresponding to simple affine roots and satisfying braid relations imposed by the following Coxeter diagram:

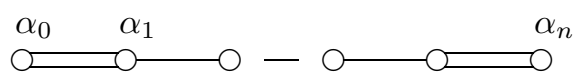

Let $l: W_{a} \rightarrow \mathbb{Z}$ be the usual length function i.e. $l(w)$ is the number of factors in a minimal expression of $w$ in terms of simple reflections. We recall the following useful fact: Let $s_{i}$ be a simple reflection. Then $l\left(s_{i} w\right)=l(w)+1$ if and only if the affine simple root $\alpha_{i}$ is positive when restricted to $w(\mathcal{C})$.

For every affine root $\beta=\alpha+m$, where $\alpha \in \Sigma$ and $m \in \mathbb{Z}$, define a map $\Phi_{\beta}: \mathrm{SL}_{2}(k) \rightarrow \operatorname{Sp}(W)$ by

$$
\Phi_{\beta}\left(\begin{array}{ll}
a & b \\
c & d
\end{array}\right)=\Phi_{\alpha}\left(\begin{array}{cc}
a & \varpi^{m} b \\
c / \varpi^{m} & d
\end{array}\right)
$$

where $\Phi_{\alpha}: \mathrm{SL}_{2}(k) \rightarrow \operatorname{Sp}(W)$ is the map defined previously for any root $\alpha \in \Sigma$. The maximal compact group $K_{i}$ is generated by $\left.\Phi_{\alpha_{j}}\left(\mathrm{SL}_{2}(R)\right)\right)$ for all affine simple roots $\alpha_{j} \neq \alpha_{i}$. For any $u$ in $k$ define

$$
x_{\beta}(u)=\Phi_{\beta}\left(\begin{array}{cc}
1 & u \\
0 & 1
\end{array}\right) .
$$

Then $U_{\beta} \cong k$ consists of all $x_{\beta}(u)$, where $u \in k$. Let $U_{\beta}(R) \cong R$ be the subgroup consisting of all $x_{\beta}(u)$ where $u \in R$. For every $t$ in $k^{\times}$we also define

$$
w_{\beta}(t)=\Phi_{\beta}\left(\begin{array}{cc}
0 & t \\
-t^{-1} & 0
\end{array}\right) \quad \text { and } \quad h_{\beta}(t)=\Phi_{\beta}\left(\begin{array}{cc}
t & 0 \\
0 & t^{-1}
\end{array}\right) .
$$

Now every element $w=s_{i_{1}} \cdots s_{i_{l}}$ in $W_{a}$ can be represented by $w_{\alpha_{i_{1}}}(1) \cdots w_{\alpha_{i_{l}}}(1)$ in $\operatorname{Sp}(W)$. Under this identification, $I$-double cosets in $\operatorname{Sp}(W)$ are represented by elements in $W_{a}$. Moreover, if $[I w I: I]$ denotes the number of single $I$-cosets in $I w I$, then $[I w I: I]=q^{l(w)}$ (see [IM]).

The length function $l$ can be generalized by giving different "weights" to simple reflections in different conjugacy classes. For example, for every positive integer $m$, we can define $l_{m}$ as follows. If $w=s_{i_{1}} \cdots s_{i_{l}}$ is a shortest expression then we define $l_{m}(w)=l_{m}\left(s_{i_{1}}\right)+\cdots+l_{m}\left(s_{i_{l}}\right)$ where $l_{m}\left(s_{i}\right)=1$ for all $i \neq n$ and $l_{m}\left(s_{n}\right)=m$. The weighted length functions naturally appear throughout the paper.

Let $J$ be an open compact subgroup defined by

$$
J=\bigcap_{i=0}^{n-1} K_{i}=I \cup I s_{n} I .
$$

Let $W_{a}^{\prime}$ be the subgroup of $W_{a}$ consisting of all elements fixing the affine root $\alpha_{n}$. This is an affine Weyl group (for $\operatorname{Sp}_{2 n-2}(k)$ ) generated by reflections $s_{0}^{\prime}=$ $s_{0}, \ldots, s_{n-2}^{\prime}=s_{n-2}$ and $s_{n-1}^{\prime}$, where $s_{n-1}^{\prime}$ corresponds to the root $\alpha_{n-1}^{\prime}=2 e_{n-1}$. Let $l^{\prime}$ be the length function on $W_{a}^{\prime}$. 
Proposition 1. Let $l$ be the length function on $W_{a}$ and $l_{3}^{\prime}$ be the length function on $W_{a}^{\prime} \subset W_{a}$ weighted so that $l_{3}^{\prime}\left(s_{n-1}^{\prime}\right)=3$ and $l_{3}^{\prime}\left(s_{i}^{\prime}\right)=1$ for $i=0, \ldots, n-2$. For every $w \in W_{a}^{\prime}$ :

(1) $l(w)=l_{3}^{\prime}(w)$.

(2) $[J w J: J]=q^{l(w)}$.

Proof. We shall prove the first statement by induction on $l^{\prime}(w)$. If $l^{\prime}(w)=0$ there is nothing to prove. Now assume that $l^{\prime}\left(s_{i}^{\prime} w\right)=l^{\prime}(w)+1$ for some simple reflection $s_{i}^{\prime}$. We need to show that $l(w)=l_{3}^{\prime}(w)$ implies $l\left(s_{i}^{\prime} w\right)=l_{3}^{\prime}\left(s_{i}^{\prime} w\right)$. Assume that $i=n-1$. Then $l_{3}^{\prime}\left(s_{n-1}^{\prime} w\right)=l_{3}^{\prime}(w)+3$. Let $x=\left(x_{1}, \ldots, x_{n}\right)$ be in the chamber $\mathcal{C}$. Then $w(x)=\left(y_{1}, \ldots, y_{n-1}, x_{n}\right)$ since $w$ is in $W_{a}^{\prime}$, and $y_{n-1}>0$ since $l^{\prime}\left(s_{n-1}^{\prime} w\right)=l^{\prime}(w)+1$. Therefore, for some $1 \leq j \leq n-1, y_{n-1}=x_{j}+m$ for an integer $m \geq 0$ or $y_{n-1}=-x_{j}+m$ for an integer $m>0$. In any case, since $\frac{1}{2}>x_{j}>x_{n}$, the inequality $y_{n-1}>x_{n}$ must hold. Thus

$$
\alpha_{n-1}(w(x))=y_{n-1}-x_{n}, \alpha_{n}\left(s_{n-1} w(x)\right)=y_{n-1}
$$

and

$$
\alpha_{n-1}\left(s_{n} s_{n-1} w(x)\right)=x_{n}+y_{n-1}
$$

are all positive and, therefore, $s_{n-1} w, s_{n} s_{n-1} w$ and $s_{n-1} s_{n} s_{n-1} w$ are the shortest expressions in $W_{a}$. Since $s_{n-1}^{\prime}=s_{n-1} s_{n} s_{n-1}$, it follows that $l\left(s_{n-1}^{\prime} w\right)=l(w)+3$, as desired. The case $i \neq n-1$ is easier, and left to the interested reader. This proves the first statement.

To prove the second statement, note that $J=I \cup U_{\alpha_{n}}(R) s_{n} I=I \cup I s_{n} U_{\alpha_{n}}(R)$. Since $w$ is in $W_{a}^{\prime}$, it commutes with $U_{\alpha_{n}}$ and $s_{n}$. Thus one easily checks that $J w J=I w I \cup I s_{n} w I$. Hence

$$
[J w J: I]=[I w I: I]+\left[I s_{n} w I: I\right]=q^{l(w)}+q^{l\left(s_{n} w\right)}=q^{l(w)}(1+q) .
$$

Since $[J: I]=1+q$ the second statement follows. The proposition is proved.

\section{Steinberg group}

Let $\pi: \widetilde{\mathrm{Sp}}(W) \rightarrow \mathrm{Sp}(W)$ be a non-trivial 2-fold central extension. In this section we describe some general properties of $\widetilde{\mathrm{Sp}}(W)$ due to Matsumoto [Ma, Moore [Mo and Steinberg [St]. Most of these properties can be directly derived in the construction of the Weil representation We. However, since the purpose of this article is not a detailed exposition, we may as well spell out some general facts first.

For every affine root $\alpha$ the central extension splits over the subgroup $U_{\alpha} \subseteq$ $\operatorname{Sp}(W)$, that is, there is a homomorphism $i_{\alpha}: U_{\alpha} \rightarrow \widetilde{\operatorname{Sp}}(W)$ such that $\pi \circ i_{\alpha}$ is the identity map on $U_{\alpha}$. The map $i_{\alpha}$ is unique since $U_{\alpha}$ is a 2-divisible group. Abusing notation, we shall consider $x_{\alpha}(u)$ as elements of $\widetilde{\mathrm{Sp}}(W)$. For any $t$ in $k^{\times}$define

$$
w_{\alpha}(t)=x_{\alpha}(t) x_{-\alpha}\left(-t^{-1}\right) x_{\alpha}(t) \quad \text { and } \quad h_{\alpha}(t)=w_{\alpha}(t) w_{\alpha}(1)^{-1} .
$$

Then, in contrast with the linear group,

$$
h_{\alpha}(t) h_{\alpha}(s)=(t, s)^{m_{\alpha}} h_{\alpha}(t s)
$$

where $(t, s)$ is the Hilbert symbol of order $2, m_{\alpha}=1$ for long roots and $m_{\alpha}=2$ for short roots. If the residual characteristic is odd then the Hilbert symbol is tame, i.e., $(s, t)=1$ for any two elements in $R^{\times}$and the central extension splits over $\Phi_{\alpha}\left(\mathrm{SL}_{2}(R)\right)$ for any affine root $\alpha$. In other words, the map $\Phi_{\alpha}$ can be lifted to

$$
\widetilde{\Phi}_{\alpha}: \mathrm{SL}_{2}(R) \rightarrow \widetilde{\operatorname{Sp}}(W) .
$$


Note that the lift $\widetilde{\Phi}_{\alpha}$ is unique. Indeed any two would have to differ by a character of $\mathrm{SL}_{2}(R)$ of order 2. But $\mathrm{SL}_{2}(R)$ has no such characters, since it is generated by pro- $p$ groups $U_{\alpha}(R)$ and $U_{-\alpha}(R)$, and $p \neq 2$. Finally, the map $\widetilde{\Phi}_{\alpha}$ can be extended to $\mathrm{SL}_{2}(k)$ if and only if $\alpha$ is a short root.

\section{Metaplectic Group}

Let $\psi$ be a non-trivial additive character of $k$. The conductor of $\psi$ is the smallest integer $c$ such that $\psi$ is trivial on $\varpi^{c} R$. Without loss of generality we may assume that $c=0$ or $c=1$. Let $H=W \times k$ be the Heisenberg group. The multiplication in $H$ is defined by

$$
\left(v_{1}, z_{1}\right) \cdot\left(v_{2}, z_{2}\right)=\left(v_{1}+v_{2},\left\langle v_{1}, v_{2}\right\rangle+z_{1}+z_{2}\right) .
$$

The center of $H$ consists of elements $(0, z)$. In particular, the center is isomorphic to $k$. There exists a unique, up to isomorphism, irreducible representation $\rho_{\psi}$ of the Heisenberg group with the central character $\psi$. If we fix a decomposition $W=X+X^{*}$ of the symplectic space $W$ as in Section 2 then $\rho_{\psi}$ can be realized on the space $S\left(X^{*}\right)$ of locally constant, compactly supported functions on $X^{*}$, such that

$$
\rho_{\psi}(x, 0) f(y)=\psi(2\langle y, x\rangle) f(y) \quad \text { and } \quad \rho_{\psi}\left(x^{*}, 0\right) f(y)=f\left(y+x^{*}\right)
$$

where $f \in S\left(X^{*}\right), x \in X$ and $x^{*} \in X^{*}$.

For every $g \in \operatorname{Sp}(W)$, the map $(v, z) \rightarrow g(v, z)=(g v, z)$ is an automorphism of $H$. Let $\rho_{\psi}^{g}$ be the representation of $H$ defined by $\rho_{\psi}^{g}(h)=\rho_{\psi}(g(h))$. Since the central character of $\rho_{\psi}^{g}$ is also $\psi$, these two representations are isomorphic. Thus, there exists $g^{\prime} \in \operatorname{GL}\left(S\left(X^{*}\right)\right)$ such that $g^{\prime} \circ \rho_{\psi}(h)=\rho_{\psi}^{g}(h) \circ g^{\prime}$ for all $h \in H$. Since $g^{\prime}$ is unique up to an element in $\mathbb{C}^{\times}$, the set of all $g^{\prime}$ for all $g \in \operatorname{Sp}(W)$ is a central extension

$$
1 \rightarrow \mathbb{C}^{\times} \rightarrow \mathrm{Sp}^{\prime}(W) \rightarrow \mathrm{Sp}(W) \rightarrow 1 .
$$

Weil has shown that the derived group of $\operatorname{Sp}(W)^{\prime}$ is isomorphic to $\widetilde{\operatorname{Sp}}(W)$, the 2-fold central extension of $\operatorname{Sp}(W)$. We shall now work out the elements $x_{\alpha}(u)$ in $\widetilde{\operatorname{Sp}}(W)$ for various roots $\alpha \in \Sigma$. Assume first that $\alpha=e_{i}-e_{j}$, a short root. Define

$$
\widetilde{\Phi}_{\alpha}: \mathrm{SL}_{2}(k) \rightarrow \mathrm{Sp}^{\prime}(W)
$$

by

$$
\left[\widetilde{\Phi}_{\alpha}(g)(f)\right](x)=f\left(g^{-1} x\right)
$$

where $f \in S\left(X^{*}\right)$, and the action of $\mathrm{SL}_{2}(k)$ on $X^{*}$ is specified in Section 2 , Since $\mathrm{SL}_{2}(k)$ is perfect, $\widetilde{\Phi}_{\alpha}\left(\mathrm{SL}_{2}(k)\right)$ is contained in $\widetilde{\mathrm{Sp}}(W)$. Thus

$$
x_{\alpha}(u)=\widetilde{\Phi}_{\alpha}\left(\begin{array}{cc}
1 & u \\
0 & 1
\end{array}\right) .
$$

Assume now that $\alpha=2 e_{i}$, a long root. Without loss of generality assume that $i=n$. Recall, from Section 2, that $W_{n-1} \subseteq W$ is the symplectic subspace spanned by $\mathbf{e}_{n}$ and $\mathbf{e}_{n}^{*}$. Let $Z_{n-1}^{*}$ be the line spanned by $\mathbf{e}_{n}^{*}$. Then $S\left(X^{*}\right)=S\left(X_{n-1}^{*}\right) \otimes S\left(Z_{n-1}^{*}\right)$ and we have an inclusion $\operatorname{Sp}^{\prime}\left(W_{n-1}\right) \subseteq \operatorname{Sp}^{\prime}(W)$ where $\operatorname{Sp}^{\prime}\left(W_{n-1}\right)$ acts on $S\left(Z_{n-1}^{*}\right)$. Thus, for ease of notation, we may assume that $W_{n-1}=W$, i.e., $\operatorname{dim}(W)=2$. Then the group $\operatorname{Sp}^{\prime}(W)$ contains operators defined by

$$
\begin{gathered}
{\left[x_{\alpha}^{\prime}(u) f\right](x)=\psi\left(u x^{2}\right) f(x),} \\
{\left[h_{\alpha}^{\prime}(t) f\right](x)=|t|^{\frac{1}{2}} f(t x),}
\end{gathered}
$$


and

$$
\left[w_{\alpha}^{\prime}(-1 / t) f\right](x)=\left[w_{-\alpha}^{\prime}(t) f\right](x)=|t|^{\frac{1}{2}} \int_{k} f(t y) \psi(2 x y) d y
$$

where $d x$ is a self-dual measure, i.e., $w_{\alpha}^{\prime}(1)^{2}=h_{\alpha}^{\prime}(-1)$. We note some obvious properties of these elements:

$$
\begin{gathered}
h_{\alpha}^{\prime}(s) h_{\alpha}^{\prime}(t)=h_{\alpha}^{\prime}(s t), \\
h_{\alpha}^{\prime}(t) x_{\alpha}^{\prime}(u) h_{\alpha}^{\prime}(t)^{-1}=x_{\alpha}^{\prime}\left(t^{2} u\right),
\end{gathered}
$$

and

$$
h_{\alpha}^{\prime}(t)=w_{\alpha}^{\prime}(t) w_{\alpha}^{\prime}(1)^{-1} .
$$

These elements differ from $x_{\alpha}(u), h_{\alpha}(t)$ and $w_{\alpha}(t)$ by non-zero complex numbers. We claim that $x_{\alpha}^{\prime}(u)=x_{\alpha}(u)$. Pick $t \in k^{\times}$such that $t^{2} \neq 1$. Since $\left[h_{\alpha}^{\prime}(t), x_{\alpha}^{\prime}(u)\right]=$ $x_{\alpha}^{\prime}\left(\left(t^{2}-1\right) u\right)$ for every $u \in k, x_{\alpha}^{\prime}(u)$ is contained in $\widetilde{\operatorname{Sp}}(W)$. Thus $x_{\alpha}(u)=x_{\alpha}^{\prime}(u)$, since the group $U_{\alpha}$ has a unique lift to $\widetilde{\operatorname{Sp}}(W)$. Moreover, since $w_{\alpha}(1)$ is a non-zero multiple of $w_{\alpha}^{\prime}(1)$, the element $x_{-\alpha}(t)$ in $\widetilde{\operatorname{Sp}}(W)$ is necessarily given by

$$
x_{-\alpha}(t)=w_{\alpha}^{\prime}(-1) x_{\alpha}(-t) w_{\alpha}^{\prime}(1) .
$$

Since $w_{\alpha}(t)=x_{\alpha}(t) x_{-\alpha}\left(-t^{-1}\right) x_{\alpha}(t)$, one can now compute $w_{\alpha}(t)$ explicitly and compare it with $w_{\alpha}^{\prime}(t)$. It turns out that

$$
w_{\alpha}(t)=\gamma\left(\psi_{t} \circ x^{2}\right) \cdot w_{\alpha}^{\prime}(t)
$$

where $\gamma\left(\psi_{t} \circ x^{2}\right)$ is the Weil index $\underline{\mathrm{Ku} 2}$ attached to the quadratic form $x^{2}$ and the character $\psi_{t}$. It is an 8-th root of 1 . It follows that $h_{\alpha}(t)=\gamma\left(t, \psi \circ x^{2}\right) h_{\alpha}^{\prime}(t)$, where $\gamma\left(t, \psi \circ x^{2}\right)=\gamma\left(\psi_{t} \circ x^{2}\right) / \gamma\left(\psi \circ x^{2}\right)$, and

$$
h_{\alpha}(s) h_{\alpha}(t)=(s, t) h_{\alpha}(s t)
$$

where $(s, t)$ is the Hilbert symbol, by the properties of the Weil index. In view of appearance of 8-th roots of one, it is natural to work with a group slightly larger than $\widetilde{\mathrm{Sp}}(W)$. Thus, let $\widetilde{\mathrm{Sp}}(W)_{8} \subseteq \mathrm{Sp}^{\prime}(W)$ be the central extension of $\operatorname{Sp}(W)$ by $\mu_{8}$, the group of 8 -th roots of one:

$$
1 \rightarrow \mu_{8} \rightarrow \widetilde{\mathrm{Sp}}(W)_{8} \rightarrow \mathrm{Sp}(W) \rightarrow 1 .
$$

An advantage of this group is that it contains the elements $h_{\alpha}^{\prime}(t)$ and $w_{\alpha}^{\prime}(t)$, defined above, for every long root $\alpha=2 e_{i}$. These elements, together with $w_{\alpha}(t)$ for short roots $\alpha=e_{i}-e_{j}$ generate a subgroup isomorphic to the normalizer of the torus $T$ in $\operatorname{Sp}(W)$.

It is useful to extend the definition of $w_{\alpha}^{\prime}(t)$ to affine roots. If $\beta=\alpha+m$ is an affine root we define $w_{\beta}^{\prime}(t)$ to be $w_{\alpha}^{\prime}\left(\varpi^{m} t\right)$. We record the following simple result which can be easily verified using the definition of $w_{\alpha}^{\prime}(t)$.

Lemma 2. Let $\alpha=2 e_{i}$ and $\beta=1-2 e_{i}$. Let $\varphi \in S\left(X^{*}\right)$ be the characteristic function of the lattice spanned by $\mathbf{e}_{1}^{*}, \ldots, \mathbf{e}_{n}^{*}$. Let $\langle\cdot, \cdot\rangle$ denote $L^{2}$-inner product on $S\left(X^{*}\right)$ with respect to the unique Haar measure on $X^{*}$ such that the Fourier transform, defined by means of $\psi$ and the Haar measure, preserves $L^{2}$-norm.

(1) If the conductor of $\psi$ is 0 then $\left\langle w_{\beta}^{\prime}(1)(\varphi), \varphi\right\rangle=q^{-\frac{1}{2}}\langle\varphi, \varphi\rangle$.

(2) If the conductor of $\psi$ is 1 then $\left\langle w_{\alpha}^{\prime}(1)(\varphi), \varphi\right\rangle=q^{-\frac{1}{2}}\langle\varphi, \varphi\rangle$. 
Recall, from Section 2, the decomposition $W=X_{l}+W_{l}+X_{l}^{*}$ and the maximal parabolic subgroup $P$ defined as the stabilizer of $X_{l}$ in $\operatorname{Sp}(W)$. Let $U_{P}$ be the unipotent radical of $P$. Then we have a Levi decomposition $P=M U_{P}$ where $M$ is chosen so that it stabilizes $X_{l}^{*}$. Let $\widetilde{P}$ the inverse image of $P$ in $\widetilde{\operatorname{Sp}}(W)_{8}$. The central extension splits uniquely over $U_{P}$, so abusing notation, we shall denote by $U_{P}$ the unipotent radical of $\widetilde{P}$. Let $\widetilde{M}$ be the inverse image of $M$ in $\widetilde{\operatorname{Sp}}(W)_{8}$. Let $Z_{l}^{*}=X^{*} \cap W_{l}$. Then $X^{*}=X_{l}^{*}+Z_{l}^{*}$. Using the decomposition $S\left(X^{*}\right)=$ $S\left(X_{l}^{*}\right) \otimes S\left(Z_{l}^{*}\right)$, we have

$$
\widetilde{M} \cong \mathrm{GL}\left(X_{l}\right) \times \widetilde{\mathrm{Sp}}\left(W_{l}\right)_{8} \cong \mathrm{GL}\left(X_{l}^{*}\right) \times \widetilde{\mathrm{Sp}}\left(W_{l}\right)_{8}
$$

where $\widetilde{\mathrm{Sp}}\left(W_{l}\right)_{8}$ acts on $S\left(Z_{l}^{*}\right)$ by the Weil representation denoted by $\omega_{\psi, l}$, and $g \in \operatorname{GL}\left(X_{l}^{*}\right)$ acts on $f \in S\left(X_{l}^{*}\right)$ by

$$
[g \cdot f](x)=|\operatorname{det}(g)|^{-\frac{1}{2}} f\left(g^{-1} x\right) .
$$

We also define $\widetilde{\mathrm{Sp}}\left(W_{n}\right)_{8}=\mu_{8}$. It acts on $S\left(Z_{n}^{*}\right) \cong \mathbb{C}$ by the character given by the inclusion $\mu_{8} \subseteq \mathbb{C}^{\times}$. The explicit action of $U_{P}$ on $S\left(X^{*}\right)$ is not important to us. However, if $S\left(X^{*}\right)_{U_{P}}$ denotes the space of $U_{P}$ co-invariants (the Jacquet functor), then $S\left(X^{*}\right)_{U_{P}} \cong S\left(Z_{l}^{*}\right)$ and the natural projection from $S\left(X^{*}\right)$ to $S\left(X^{*}\right)_{U_{P}}$ is given by the restriction of functions from $X^{*}$ to $Z_{l}^{*}$. In particular, $g \in \mathrm{GL}\left(X_{l}^{*}\right)$ acts on $S\left(X^{*}\right)_{U_{P}}$ by multiplication by $|\operatorname{det}(g)|^{1 / 2}$.

\section{Minimal types in Weil Representation}

Recall that $K_{i}$ is the maximal open compact subgroup defined as the stabilizer of the good lattice $L_{i}$, where $L_{i}$ is the $R$-span of $\mathbf{e}_{j}$ for $1 \leq j \leq n$, $\frac{\mathbf{e}_{j}^{*}}{\varpi}$ for $j \leq$ $i$, and $\mathbf{e}_{j}^{*}$ for $j>i$. Then $K_{i}$ contains a pro- $p$ subgroup such that $K_{i} / K_{i}^{+} \cong$ $\mathrm{Sp}_{2 i}(\mathfrak{f}) \times \operatorname{Sp}_{2(n-i)}(\mathfrak{f})$. Let $\tilde{K}_{i}$ be the inverse image of $K_{i}$ in $\widetilde{\operatorname{Sp}}(W)_{8}$. We shall see that $\tilde{K}_{i} \rightarrow K_{i}$ splits, but first, we describe the minimal $\tilde{K}_{i}$-types in $\omega_{\psi}$, the Weil representation of $\widetilde{\operatorname{Sp}}(W)_{8}$, realized on $S\left(X^{*}\right)$.

Case (i): conductor of $\psi$ is 0 . In this case there is a $\tilde{K}_{i}$-invariant submodule $\tau_{i}$ consisting of functions supported on $L_{i} \cap X^{*}$ and invariant under translations by the lattice $L_{0} \cap X^{*}$. Thus this space can be written as

$$
\tau_{i} \cong S\left(L_{i} / L_{0}\right) \otimes \mathbb{C}
$$

where $S\left(L_{i} / L_{0}\right)$ is naturally the Weil representation of $\mathrm{Sp}_{2 i}(\mathfrak{f})$ corresponding to the character of $\mathfrak{f} \cong \frac{1}{\varpi} R / R$ obtained by restricting $\psi$ to $\frac{1}{\varpi} R$, and $\mathbb{C}$ is the trivial representation of $\operatorname{Sp}_{2(n-i)}(\mathfrak{f})$. Note that $\tau_{0}$ is one-dimensional and spanned by $\varphi$, the characteristic function of $L_{0} \cap X^{*}$. If $i \neq 0$, then $\tau_{i}=\tau_{i}^{e} \oplus \tau_{i}^{o}$ is a sum of even and odd Weil representations of $\operatorname{Sp}_{2 i}(\mathfrak{f})$. Note that we have a natural inclusion of spaces $\tau_{i} \subseteq \tau_{i+1}$ for all $i<n$.

Case (ii): conductor of $\psi$ is 1 . In this case there is a $\tilde{K}_{i}$-invariant submodule $\tau_{i}$ consisting of functions supported on $L_{0} \cap X^{*}$ and invariant under translations by the lattice $\varpi L_{i} \cap X^{*}$. Thus this space can be written as

$$
\tau_{i} \cong \mathbb{C} \otimes S\left(L_{0} / \varpi L_{i}\right)
$$

where $S\left(L_{0} / \varpi L_{i}\right)$ is naturally the Weil representation of $\operatorname{Sp}_{2(n-i)}(\mathfrak{f})$ corresponding to the character of $\mathfrak{f} \cong R / \varpi R$ obtained by restricting $\psi$ to $R$, and $\mathbb{C}$ is the trivial representation of $\operatorname{Sp}_{2 i}(\mathfrak{f})$. Note that $\tau_{n}$ is one-dimensional and spanned by $\varphi$, the 
characteristic function of $L_{0} \cap X^{*}$. If $i \neq n$, then $\tau_{i}=\tau_{i}^{e} \oplus \tau_{i}^{o}$ is a sum of even and odd Weil representations of $\operatorname{Sp}_{2(n-i)}(\mathfrak{f})$. Note that we have a natural inclusion of spaces $\tau_{i+1} \subseteq \tau_{i}$ for all $i<n$.

Now we can prove that the central extension $\tilde{K}_{i} \rightarrow K_{i}$ splits for all $i$. Let $d_{e}$ and $d_{o}$ be the determinant characters of $\tilde{K}_{i}$ acting on $\tau_{i}^{e}$ and $\tau_{i}^{o}$, respectively. Since $\operatorname{dim} \tau_{i}^{e}-\operatorname{dim} \tau_{i}^{o}=1$, the character

$$
d_{e}^{\operatorname{dim} \tau_{i}^{e}} \cdot d_{o}^{-\operatorname{dim} \tau_{i}^{e}}: \tilde{K}_{i} \rightarrow \mu_{8}
$$

is the identity on $\mu_{8} \subseteq \tilde{K}_{i}$.

\section{Preliminaries on Hecke algebras}

Let $G$ be a totally disconnected topological group and $K$ an open compact subgroup. Fix a measure on $G$ such that the volume of $K$ is one. Let $(\sigma, E)$ be an irreducible, finite dimensional representation of $K$. Let $\left(\sigma^{*}, E^{*}\right)$ be the dual representation. Let $H(\sigma)$ be the space of locally constant, compactly supported functions

$$
f: G \rightarrow \operatorname{End}\left(E^{*}\right)
$$

such that

$$
f\left(k_{1} g k_{2}\right)=\sigma^{*}\left(k_{1}\right) f(g) \sigma^{*}\left(k_{2}\right)
$$

for all $k_{1}, k_{2} \in K$. This is an algebra with respect to the convolution of functions. For any smooth representation $(\pi, V)$ of $G$, the space $\left(V \otimes E^{*}\right)^{K}$ is an $H(\sigma)$-module, where the action of $f \in H(\sigma)$ is defined by

$$
\pi(f)(v \otimes e)=\int_{G} \pi(g) v \otimes f(g) e d g .
$$

Let $\Gamma$ be an open compact subgroup of $G$ containing $K$. Then $K$ has finite index in $\Gamma$. Let $H_{\Gamma}(\sigma)$ be the subalgebra of $H(\sigma)$ consisting of functions supported on $\Gamma$. Then $H_{\Gamma}(\sigma)$ is finite dimensional and it is isomorphic to the algebra of intertwining operators of the representation

$$
\operatorname{Ind}_{K}^{\Gamma}\left(\sigma^{*}\right)=\left\{\phi: \Gamma \rightarrow E^{*}: \phi(k g)=\sigma^{*}(k) \phi(g) \quad \text { for all } k \in K, g \in \Gamma\right\} .
$$

Indeed, for $f \in H_{\Gamma}(\sigma)$ and $\phi \in \operatorname{Ind}_{K}^{\Gamma}\left(\sigma^{*}\right)$, let

$$
(L(f) \phi)(g)=\int_{\Gamma} f(h) \phi\left(h^{-1} g\right) d h \quad \text { for all } g \in \Gamma .
$$

The map $f \mapsto L(f)$ gives an isomorphism of $H_{\Gamma}(\sigma)$ and the endomorphism algebra of $\operatorname{Ind}_{K}^{\Gamma}\left(\sigma^{*}\right)$. Assume now that $H_{\Gamma}(\sigma)$ is commutative. Let $V$ be an irreducible finite dimensional representation of $\Gamma$ such that $\left(V \otimes E^{*}\right)^{K} \neq 0$. Then, since $H_{\Gamma}(\sigma)$ is commutative, $\left(V \otimes E^{*}\right)^{K}$ is one-dimensional and the action of $H_{\Gamma}(\sigma)$ on it factors through a maximal ideal $m \subseteq H_{\Gamma}(\sigma)$. Moreover,

$$
\operatorname{Ind}_{K}^{\Gamma}\left(\sigma^{*}\right) / L(m) \cdot \operatorname{Ind}_{K}^{\Gamma}\left(\sigma^{*}\right) \cong V^{*}
$$

the dual representation of $V$. Thus, if $\left(\pi_{1}, V_{1}\right), \ldots,\left(\pi_{l}, V_{l}\right)$ are all, up to isomorphism, irreducible representations of $\Gamma$ such that $\left(V \otimes E^{*}\right)^{K} \neq 0$, then

$$
\operatorname{Ind}_{K}^{\Gamma}\left(\sigma^{*}\right) \cong V_{1}^{*} \oplus \ldots \oplus V_{l}^{*} \text {. }
$$

Moreover, for every $f \in H_{\Gamma}(\sigma)$, Trace $(L(f))=\sum_{i=1}^{l} d_{i} \lambda_{i}$ where $d_{i}$ is the dimension of $V_{i}$ and $\lambda_{i}=\pi_{i}(f)$. Note that, if $f$ is not supported on $K$, then the trace of $L(f)$ is 0 . Indeed, if $\Gamma=\bigcup_{i=1}^{r} K g_{i}$, then $\operatorname{Ind}_{K}^{\Gamma}\left(\sigma^{*}\right)$ has a basis $\phi_{i}$ of functions supported 
on $K g_{i}$. If $f$ is supported on a double coset $K \gamma K=\bigcup_{j=1}^{s} K \gamma_{j}$, then $L(f)\left(\phi_{i}\right)$ is supported on $\bigcup_{j=1}^{s} K \gamma_{j} g_{i}$ and this does not contain $K g_{i}$ if $K \gamma K \neq K$.

Thus we have proven:

Lemma 3. Assume that the dimension of $H_{\Gamma}(\sigma)$ is two. Let $\left(\pi_{1}, V_{1}\right)$ and $\left(\pi_{2}, V_{2}\right)$ be the unique, up to isomorphism, irreducible representations of $\Gamma$ such that $\left(V \otimes E^{*}\right)^{K} \neq 0$. Let $d_{1}$ and $d_{2}$ be their dimensions. Let $T$ be an element in $H_{\Gamma}(\sigma)$ not supported on $K$. Let $\lambda_{1}=\pi_{1}(T)$ and $\lambda_{2}=\pi_{2}(T)$. Then

(1) $\operatorname{dim}\left(\operatorname{Ind}_{K}^{\Gamma}\left(\sigma^{*}\right)\right)=d_{1}+d_{2}$.

(2) $d_{1} \lambda_{1}+d_{2} \lambda_{2}=0$.

(3) The minimal polynomial of $T$ is $\left(T-\lambda_{1}\right)\left(T-\lambda_{2}\right)$.

\section{Hecke algebra $H_{\psi}^{+}$}

Let $I=\bigcap_{i=0}^{n} K_{i}$ be the Iwahori subgroup of $\operatorname{Sp}(W)$, and let $\tilde{I}$ be the inverse image of $I$ in $\widetilde{\operatorname{Sp}}(W)_{8}$. The Hecke algebra $H_{\psi}^{+}$, we are about to define, depends on $\psi$. We have two cases to consider. Assume first that the conductor of $\psi$ is 0 . Let $\tau_{0} \subseteq S\left(X^{*}\right)$ be the one-dimensional minimal $\tilde{K}_{0}$-type. Let $H_{\psi}^{+}=H\left(\tau_{0}\right)$ be the Hecke algebra, defined as in Section 7 , for $G=\widetilde{\operatorname{Sp}}(W)_{8}, K=\tilde{I}$ and $\sigma=\tau_{0}$. Since $\tau_{0}$ is one-dimensional, some definitions from Section 7 simplify in this case. First, since $\operatorname{End}\left(\tau_{0}^{*}\right) \cong \mathbb{C}$, the algebra $H_{\psi}^{+}$consists of complex valued functions. Second, if $(\pi, V)$ is a smooth representation of $\widetilde{\mathrm{Sp}}(W)_{8}$, the Hecke module $\left(V \otimes \tau_{0}^{*}\right)^{\tilde{I}}$ can be identified with

$$
V^{\tilde{I}, \tau_{0}}=\left\{v \in V \mid \pi(i) v=\tau_{0}(i) v \text { for all } i \in \tilde{I}\right\} .
$$

We shall now determine the algebra $H_{\psi}^{+}$in terms of its generator and relations. If for some $x$ there exists $i_{1}, i_{2} \in \tilde{I}$ such that $i_{1} x=x i_{2}$ and $\tau_{0}^{*}\left(i_{1}\right) \neq \tau_{0}^{*}\left(i_{2}\right)$ then $f(x)=0$ for any $f$ in the Hecke algebra. Thus, it is not a priori clear that every $\tilde{I}$-double coset supports an element in the Hecke algebra. We address this delicate issue as follows. For any simple affine root $\alpha_{i}$, define an open compact subgroup

$$
\tilde{I}_{i}=\tilde{I} \cup \tilde{I} w_{\alpha_{i}}(1) \tilde{I} .
$$

Let $H_{\psi, i}$ be the subalgebra of $H_{\psi}^{+}$consisting of functions supported on $\tilde{I}_{i}$. This is a one or two-dimensional subalgebra spanned by the identity element of $H_{\psi}^{+}$and an element $T_{i}$ supported on $\tilde{I} w_{\alpha_{i}}(1) \tilde{I}$ if such an element exists. Recall, from Section 7 . that the algebra $H_{\psi, i}$ is naturally isomorphic to the endomorphism algebra of the induced representation $\operatorname{Ind}_{\tilde{I}}^{\tilde{I}_{i}}\left(\tau_{0}^{*}\right)$, of dimension $q+1$. Thus, in order to show that $T_{i}$ exists, it suffices to show that this representation is reducible. Since $\operatorname{Ind}_{\tilde{I}} \tilde{I}_{i}\left(\tau_{0}^{*}\right)$ is dual to $\operatorname{Ind}_{\tilde{I}}^{\tilde{I}_{i}}\left(\tau_{0}\right)$, it suffices to show that $\operatorname{Ind}_{\tilde{I}}^{\tilde{I}_{i}}\left(\tau_{0}\right)$ is reducible. Assume first that $i \neq 0$. Then $\tilde{I}_{i} \subseteq \tilde{K}_{0}$ so $\tau_{0} \subseteq S\left(X^{*}\right)$ is naturally a representation of $\tilde{I}_{i}$. Thus, by the Frobenius reciprocity, $\tau_{0}$ is a one-dimensional summand of $\operatorname{Ind}_{\tilde{I}} \tilde{I}_{i}\left(\tau_{0}\right)$. Assume now $i=0$. In this case $\tilde{I}_{0} \subseteq \tilde{K}_{1}$. Consider the $\tilde{K}_{1}$-type $\tau_{1} \subseteq S\left(X^{*}\right)$. Then $\tau_{1}=\tau_{1}^{e} \oplus \tau_{1}^{o}$ is a sum of an even and odd Weil representation of $\mathrm{SL}_{2}(\mathfrak{f})$. Since $\tau_{0} \subseteq \tau_{1}^{e}$, the Frobenius reciprocity implies that $\tau_{1}^{e}$ is a summand of $\operatorname{Ind}_{\tilde{I}} \tilde{I}_{i}\left(\tau_{0}\right)$ of dimension $(q+1) / 2$. We have now shown that $T_{i}$ exists and we normalize it so that $T_{i}\left(w_{\alpha_{i}}(1)\right)=1$ if $i \neq 0, n, T_{0}\left(w_{\alpha_{0}}^{\prime}(1)\right)=q^{-\frac{1}{2}}$, and $T_{n}\left(w_{\alpha_{n}}^{\prime}(1)\right)=1$. 
Lemma 4. Let $\omega_{\psi}$ denote the action of $\widetilde{\operatorname{Sp}}(W)_{8}$ on $S\left(X^{*}\right)$. Let $\varphi \in \tau_{0} \subseteq S\left(X^{*}\right)$ be the characteristic function of the lattice $L_{0} \cap X^{*}$. Then $\omega_{\psi}\left(T_{i}\right) \varphi=q \varphi$ if $i \neq 0$ and $\omega_{\psi}\left(T_{0}\right) \varphi=\varphi$.

Proof. The idea is to compute the inner product $\left\langle\omega_{\psi}\left(T_{i}\right) \varphi, \varphi\right\rangle$. Let $I^{+}$be the pro- $p$ radical of $\tilde{I}$. Then $\tilde{I} w_{\alpha_{i}}(1) \tilde{I}=I^{+} w_{\alpha_{i}}(1) \tilde{I}$, so we can write

$$
\tilde{I} w_{\alpha_{i}}(1) \tilde{I}=\bigcup_{j=1}^{q} x_{j} w_{\alpha}(1) \tilde{I}
$$

for some elements $x_{j}$ in $I^{+}$. Assume now that $i \neq 0, n$. Then

$$
\left\langle\omega_{\psi}\left(T_{i}\right) \varphi, \varphi\right\rangle=\sum_{j=1}^{q}\left\langle x_{j} w_{\alpha_{i}}(1) \varphi, \varphi\right\rangle=\sum_{j=1}^{q}\left\langle w_{\alpha_{i}}(1) \varphi, x_{j}^{-1} \varphi\right\rangle=q\langle\varphi, \varphi\rangle
$$

where the last equality holds since $x_{j}$ and $w_{\alpha_{i}}(1)$ fix $\varphi$. A similar argument works for $i=n$, since $w_{\alpha_{n}}^{\prime}(1)$ is the Fourier transform in the variable $x_{n}$ and it fixes $\varphi$, since the conductor is 0. For the case $i=0$, use Lemma 2, part (1).

Lemma 5. The operator $T_{i}$ satisfies the quadratic relations $\left(T_{i}-q\right)\left(T_{i}+1\right)=0$ if $i \neq 0$ and $T_{0}^{2}=1$. In particular, all $T_{i}$ are invertible.

Proof. This follows easily from Lemma 3. Assume first that $i \neq 0$. Then $\tau_{0}$ is a one-dimensional summand of $\operatorname{Ind}_{\tilde{I}}^{\tilde{I}_{i}}\left(\tau_{0}\right)$. Thus $d_{1}=1$ and $d_{2}=q$. Lemma 4 implies that $\lambda_{1}=q$. It follows that $\lambda_{2}=-1$, by Lemma 3 (2). Assume now $i=0$. In this case $\tau_{1}^{e}$ is a summand of $\operatorname{Ind}_{\tilde{I}}^{\tilde{I}_{i}}\left(\tau_{0}\right)$ of dimension $(q+1) / 2$. Thus $d_{1}=d_{2}=(q+1) / 2$. Since $\left(\tau_{1}^{e}\right)^{\tilde{I}, \tau_{0}}=\tau_{0}$, Lemma 4 implies that $\lambda_{1}=1$. It follows that $\lambda_{2}=-1$, by Lemma 3 (2). Finally, invertibility of $T_{i}$ follows from the quadratic relations. For example, if $i \neq 0$, then $q T_{i}^{-1}=T_{i}-q+1$. The lemma is proved.

Theorem 6. Assume that the conductor of $\psi$ is 0 . The Hecke algebra $H_{\psi}^{+}$, as an abstract algebra, is generated by elements $T_{0}, T_{1}, \ldots, T_{n}$ satisfying quadratic relations $\left(T_{i}-q\right)\left(T_{i}+1\right)=0$ for $i \neq 0, T_{0}^{2}=1$, and satisfying the braid relations corresponding to the following Coxeter diagram:

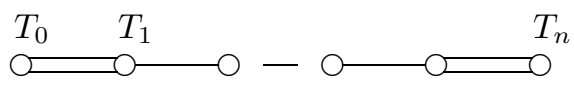

Proof. We have already shown that the quadratic relations hold. Thus, we need to prove braid relations. Since $s_{0} s_{1} s_{0} s_{1}=s_{1} s_{0} s_{1} s_{0}$ (a braid relation in the affine Weyl group) it follows that $T_{0} T_{1} T_{0} T_{1}$ and $T_{1} T_{0} T_{1} T_{0}$ are supported on the same double coset. Thus they must be equal up to a scalar multiple. However, by Lemma 4 both operators act on the type $\tau_{0}$ by the same positive scalar. Thus they are equal. Other braid relations are, of course, proved in the same manner. The rest of the proof is completely analogous to the case of linear groups.

If the conductor of $\psi$ is 1 , then the role of $K_{0}$ is played by $K_{n}$. We define $H_{\psi}^{+}=H\left(\tau_{n}\right)$ where $\tau_{n}$ is the one-dimensional $\tilde{K}_{n}$-type, viewed as a representation of $\tilde{I}$. An obvious analogue of Theorem 6 holds. 


\section{BeRnstein's COMPONENT $\mathcal{G}_{\psi}^{+}$}

In this section we use a decomposition of the category of genuine representations of $\widetilde{\mathrm{Sp}}(W)$ given by Bernstein Be. As indicated there, the decomposition is also valid for central extensions. In fact, the modifications of arguments for central extensions (2.2 in $[\overline{\mathrm{Be}})$ are trivial for $\widetilde{\mathrm{Sp}}(W)$, since $\tilde{T}$ is commutative.

Let $\widetilde{B}=\widetilde{T} U \subseteq \widetilde{\mathrm{Sp}}(W)_{8}$ be the inverse image of the Borel subgroup defined in Section 2. For any $\alpha=2 e_{i}$ and $t \in k^{\times}$, let us abbreviate $h_{i}^{\prime}(t)=h_{\alpha}^{\prime}(t)$. Any element $t \in \widetilde{T}$ can be uniquely written as

$$
t=h_{1}^{\prime}\left(t_{1}\right) \cdot \ldots \cdot h_{n}^{\prime}\left(t_{n}\right) \cdot \zeta
$$

where $t_{i} \in k^{\times}$and $\zeta \in \mu_{8}$. Then any $n$-tuple of characters $\chi=\left(\chi_{1}, \ldots, \chi_{n}\right)$ defines a genuine character $\chi: \widetilde{T} \rightarrow \mathbb{C}^{\times}$

$$
\chi(t)=\chi_{1}\left(t_{1}\right) \cdot \ldots \cdot \chi_{n}\left(t_{n}\right) \cdot \zeta .
$$

Let $I(\chi)$ denote the normalized principal series representation, i.e., we induce the character $\chi$ multiplied by the modular character $\rho_{U}^{\frac{1}{2}}$. If $(\pi, V)$ is a smooth representation of $\widetilde{\operatorname{Sp}}\left(W_{8}\right)$, let $\left(\pi_{U}, V_{U}\right)$ denote the normalized Jacquet module, i.e., the action of $\widetilde{T}$ on $V_{U}$ is multiplied by the inverse of the modular character. With these conventions the Frobenius reciprocity takes the form

$$
\operatorname{Hom}_{\widetilde{\mathrm{Sp}}(W)_{8}}(V, I(\chi)) \cong \operatorname{Hom}_{\widetilde{T}}\left(V_{U}, \chi\right) .
$$

Let $\mathcal{G}_{\psi}^{+}$be the component of the category of smooth representations of $\widetilde{\operatorname{Sp}}(W)_{8}$ whose irreducible representation are submodules of $I(\chi)$ where $\chi$ is unramified. The even Weil representation $\omega_{\psi}^{e}$ belongs to $\mathcal{G}_{\psi}^{+}$. Indeed, the functional $\ell: S\left(X^{*}\right)^{e} \rightarrow \mathbb{C}$ defined by $\ell(f)=f(0)$, via the Frobenius reciprocity, gives an embedding of $\omega_{\psi}^{e}$ into $I\left(\chi_{e}\right)$, where $\chi_{e}$ is an unramified character.

Let $\tilde{I}_{T}=\tilde{I} \cap \widetilde{T}$, and consider $\tau_{0}$ as a character of $\tilde{I}_{T}$ by restriction. By Proposition 3.5.2 in $\mathrm{Be}$ the natural projection from $V$ to $V_{U}$ gives a surjection

$$
r: V^{\tilde{I}, \tau_{0}} \rightarrow\left(V_{U}\right)^{\tilde{I}_{T}, \tau_{0}} .
$$

For any $w \in W_{a}$, let $T_{w}=T_{i_{1}} \cdots T_{i_{l}}$ where $w=s_{i_{1}} \cdots s_{i_{l}}$, is a shortest product of simple reflections. Note that $T_{w}$ is invertible, since all $T_{i}$ are. Let $\lambda=\left(\lambda_{1}, \ldots, \lambda_{n}\right) \in$ $\mathbb{Z}^{n}$ be a translation in $W_{a}$ such that $\lambda_{1} \geq \ldots \geq \lambda_{n} \geq 0$, and let

$$
\hat{T}_{\lambda}=q^{-\frac{l_{0}(\lambda)}{2}} T_{\lambda}
$$

where $l_{0}$ is the weighted length function on $W_{a}$ such that $l_{0}\left(s_{0}\right)=0$ and 1 for other simple reflections. Let $t_{\lambda}=h_{1}^{\prime}\left(\varpi^{\lambda_{1}}\right) \cdot \ldots \cdot h_{n}^{\prime}\left(\varpi^{\lambda_{n}}\right)$. Note that, since $T_{0}\left(w_{\alpha_{0}}^{\prime}(1)\right)=$ $q^{-\frac{1}{2}}$, the value of $\hat{T}_{\lambda}$ at $t_{\lambda}$ is $q^{-\frac{l(\lambda)}{2}}$. Since

$$
\tilde{I} t_{\lambda} \tilde{I}=\bigcup_{i=1}^{q^{l(\lambda)}} t_{\lambda} u_{i} \tilde{I}
$$

where $u_{i}$ are representatives of $\tilde{I} \cap U$-co sets in $t_{\lambda}^{-1}(\tilde{I} \cap U) t_{\lambda}$, it follows that

$$
r \circ \pi\left(\hat{T}_{\lambda}\right)=\pi_{U}\left(t_{\lambda}\right) \circ r .
$$


Let $v$ be in the kernel of $r$. Then $\int_{U_{c}} \pi(u) v d u=0$ for some open compact subgroup $U_{c}$ of $U$. Pick $\lambda$ such that $t_{\lambda}^{-1}(\tilde{I} \cap U) t_{\lambda} \supseteq U_{c}$. Then

$$
\pi\left(T_{\lambda}\right)(v)=\pi\left(t_{\lambda}\right) \sum_{i=1}^{q^{l(\lambda)}} \pi\left(u_{i}\right)(v)=\int_{t_{\lambda}^{-1}(\tilde{I} \cap U) t_{\lambda}} \pi(u) v d u=0,
$$

hence $v=0$, since $T_{\lambda}$ is invertible. Thus the map $r$ is an isomorphism. In particular, if $V$ is an irreducible representation such that $V^{\tilde{I}, \tau_{0}} \neq 0$, then $V$ is a submodule of $I(\chi)$ for some character $\chi$. Moreover, $\chi$ is unramified since the character $\tau_{0}$ is trivial on $h_{i}^{\prime}(t)$ for $t \in R^{\times}$(as these elements fix the vector $\varphi \in \tau_{0}$ ). Conversely, if $V$ is a submodule of $I(\chi)$ for some unramified character $\chi$ then $\left(V_{U}\right)^{\tilde{I}_{T}, \tau_{0}} \neq 0$ and, therefore, $V^{\tilde{I}, \tau_{0}} \neq 0$. Thus irreducible representations of $H_{\psi}^{+}$correspond to irreducible constituents of principal series representations $I(\chi)$. This proves (see (3.12) in $[\mathrm{BK}]$ :

Corollary 7. The functor that attaches to every smooth representation $V$ of $\widetilde{\operatorname{Sp}}(W)_{8}$ the Hecke algebra module $V^{\tilde{I}, \tau_{0}}$ is an equivalence of Bernstein's component $\mathcal{G}_{\psi}^{+}$and the category of $\mathrm{H}_{\psi}^{+}$-modules.

Operators $\hat{T}_{\lambda}$ form a commuting family of operators. Thus any finite dimensional representation $\mathrm{F}$ of $\mathrm{H}_{\psi}^{+}$can be decomposed into a sum of generalized eigenspaces. More precisely, for any $\mu=\left(\mu_{1}, \ldots, \mu_{n}\right) \in \mathbb{C}^{n}$, define $F_{\mu}$ to be the subspace of $F$ consisting of all $v \in F$ such that

$$
\pi\left(\hat{T}_{\lambda}-q^{-\sum_{i=1}^{n} \lambda_{i} \mu_{i}}\right)^{\operatorname{dim}(F)}(v)=0 .
$$

Then $F=\bigoplus F_{\mu}$ and any $\mu$ such that $F_{\mu} \neq 0$ is called an exponent of $F$. The representation $F$ is square integrable if

$$
\Re\left(\mu_{1}\right)>0, \Re\left(\mu_{1}\right)+\Re\left(\mu_{2}\right)>0, \ldots, \Re\left(\mu_{1}\right)+\ldots+\Re\left(\mu_{n}\right)>0
$$

for all exponents $\mu$. The representation $F$ is tempered if the exponents satisfy the inequalities where all $>$ have been replaced by $\geq$. These definitions agree with the usual definitions of square integrable and tempered representations of $\widetilde{\operatorname{Sp}}(W)_{8}$, by Casselman's criterion.

Hecke algebra functors. Much of what we have discussed in this section can be generalized as follows. Let $\widetilde{P}=\widetilde{M} U_{P}$ be a parabolic subgroup of $\widetilde{\operatorname{Sp}}(W)_{8}$ in a standard position. Let $\Delta_{M} \subseteq \Delta$ be the set of simple roots of $\widetilde{M}$. Let $\tilde{I}_{M}=$ $\tilde{I} \cap \widetilde{M}$, and consider $\tau_{0}$ as a character of $\tilde{I}_{M}$. We can define a Hecke algebra $H_{\psi}^{M,+}=H\left(\tau_{0}\right)$ on $\widetilde{M}$. In particular, for every simple root $\alpha_{i} \in \Delta_{M}$ we have an element $T_{i}^{M}$ satisfying the quadratic relation $\left(T_{i}^{M}-q\right)\left(T_{i}^{M}+1\right)=0$ and for every $\lambda=\left(\lambda_{1}, \ldots, \lambda_{n}\right) \in \mathbb{Z}^{n}$ such that $\lambda_{1} \geq \ldots \geq \lambda_{n}$ we have an element $\hat{T}_{\lambda}^{M}$ whose value at $t_{\lambda}$ is $q^{-\frac{l_{M}(\lambda)}{2}}$. (Here $l_{M}$ is the length function for the affine Weyl group of M.) It follows from Bernstein's relations that there is a unique homomorphism of algebras,

$$
i_{P}: H_{\psi}^{M,+} \rightarrow H_{\psi}^{+}
$$

such that $i_{P}\left(T_{i}^{M}\right)=T_{i}$ and $i_{P}\left(\hat{T}_{\lambda}^{M}\right)=\hat{T}_{\lambda}$. Let $(\pi, V)$ be a smooth representation of $\widetilde{\mathrm{Sp}}(W)_{8}$. Then the natural projection from $V$ to $V_{U_{P}}$ (a normalized Jacquet 
functor) induces an isomorphism

$$
r_{U_{P}}: V^{\tilde{I}, \tau_{0}} \rightarrow\left(V_{U_{P}}\right)^{\tilde{I}_{M}, \tau_{0}} .
$$

One easily sees, by checking on $T_{i}^{M}$ and $\hat{T}_{\lambda}^{M}$, that $r_{U_{P}} \circ \pi\left(i_{P}(T)\right)=\pi_{U_{P}}(T) \circ$ $r_{U_{P}}$ for all $T$ in $H_{\psi}^{M,+}$. In other words, the Jacquet functor for the parabolic group $\widetilde{P}$ corresponds to the natural (forgetful) functor from $H_{\psi}^{+}$-modules to $H_{\psi}^{M,{ }^{+}}$modules. On the other hand, tensoring of $H_{\psi}^{M,+}$-modules with $H_{\psi}^{+}$correspond to the induction with respect to the parabolic group opposite to $\widetilde{P}$. For a detailed treatment of this topic the reader can consult [BK] and [Ro.

\section{Hecke algebra of $\mathrm{SO}\left(V^{+}\right)$}

The affine Hecke algebra of $\mathrm{SO}\left(V^{+}\right)$is described in terms of the extended affine Weyl group. To that end, let $\mathbb{E}=\mathbb{R} e_{1}+\ldots+\mathbb{R} e_{n}$, and let $\Sigma=\left\{ \pm e_{i} \pm e_{j}\right\} \cup\left\{ \pm e_{i}\right\}$ be a root system of type $\mathrm{B}_{n}$, sitting in $\mathbb{E}$. Fix a set $\Delta$ of simple roots consisting of roots $\alpha_{1}=e_{1}-e_{2}, \ldots, \alpha_{n-1}=e_{n-1}-e_{n}$ and $\alpha_{n}=e_{n}$. Roots can be viewed as functionals on $\mathbb{E}$, using the standard dot product on $\mathbb{E}$. Affine roots are functionals $\alpha+m$ where $\alpha \in \Sigma$ and $m \in \mathbb{Z}$. We have a set of simple affine roots $\Delta_{a}=\Delta \cup\left\{\alpha_{0}\right\}$ where $\alpha_{0}=1-e_{1}-e_{2}$. The affine Weyl group (of type $\mathrm{B}_{n}$ ) is generated by reflections $s_{0}^{\prime}$ and $s_{1}, \ldots, s_{n}$ corresponding to the simple affine roots satisfying the braid relations given by the following Coxeter diagram:

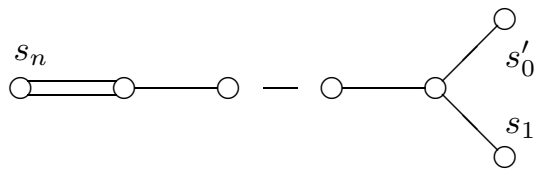

The affine Weyl group is a semi-direct product of the Weyl group (generated by $\left.s_{1}, \ldots, s_{n}\right)$ and a normal subgroup consisting of translations by $\lambda=\left(m_{1}, \ldots, m_{n}\right) \in$ $\mathbb{Z}^{n}$ such that $\sum_{i=1}^{n} m_{i}$ is even. The extended affine Weyl group, relevant for the adjoint group $\mathrm{SO}\left(V^{+}\right)$, contains one additional element $s_{0}$ such that $s_{0}^{2}=1$ and

$$
s_{0}^{\prime}=s_{0} s_{1} s_{0} .
$$

In fact, $s_{0}$ is the reflection about the hyperplane $x_{1}=\frac{1}{2}$. This shows that the extended affine Weyl group of type $\mathrm{B}_{n}$ coincides with the affine Weyl group of type $\mathrm{C}_{n}$, denoted by $W_{a}$. In particular, it is generated by reflections $s_{0}, \ldots, s_{n}$ and the braid relations corresponding to the following Coxeter diagram:

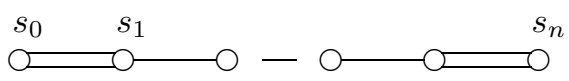

Let $l_{0}$ be the weighted length function on $W_{a}$ such that $l_{0}\left(s_{0}\right)=0$ and it is 1 on other simple reflections. Let $I$ be the Iwahori subgroup of $\mathrm{SO}\left(V^{+}\right)$. Then for every $w$ in the extended affine Weyl group $[I w I: I]=q^{l_{0}(w)}$.

Let $H^{+}$denote the affine Hecke algebra of $\mathrm{SO}\left(V^{+}\right)$. For any $w \in W_{a}$ let $T_{w} \in$ $H^{+}$be the characteristic function of the double coset parameterized by $w$. For simplicity, let $T_{i}$ denote $T_{s_{i}}$. Then $T_{i}$ satisfies $T_{0}^{2}=1$ and $\left(T_{i}-q\right)\left(T_{i}+1\right)=0$, if $i \neq 0$, and the braid relations given by the above Coxeter diagram. In fact, as an 
abstract algebra, $H^{+}$is generated by $T_{0}, \ldots, T_{n}$ modulo these quadratic and the braid relations. Since $H$ and $H_{\psi}^{+}$are given by "identical" combinatorial data (see Theorem [6), we have obtained the following:

Theorem 8. The algebra $H_{\psi}^{+}$is isomorphic, via an explicit isomorphism, to the algebra $H^{+}$.

Let $\lambda=\left(\lambda_{1}, \ldots, \lambda_{n}\right) \in \mathbb{Z}^{n}$ be a translation in $W_{a}$ such that $\lambda_{1} \geq \ldots \geq \lambda_{n} \geq 0$. Define $\hat{T}_{\lambda}=q^{-\frac{l_{0}(\lambda)}{2}} T_{\lambda}$. The operators $\hat{T}_{\lambda}$ form a commuting family of operators and, as we discussed in the previous section, any finite dimensional representation $H^{+}$can be decomposed in a sum of generalized eigenspaces, parameterized by exponents $\mu=\left(\mu_{1}, \ldots, \mu_{n}\right) \in \mathbb{C}^{n}$. Again, the corresponding representation of $\mathrm{SO}\left(V^{+}\right)$is square integrable if and only if

$$
\Re\left(\mu_{1}\right)>0, \Re\left(\mu_{1}\right)+\Re\left(\mu_{2}\right)>0, \ldots, \Re\left(\mu_{1}\right)+\ldots+\Re\left(\mu_{n}\right)>0
$$

for all exponents $\mu$, and it is tempered if the exponents satisfy the inequalities where all $>$ have been replaced by $\geq$.

The trivial representation of $\mathrm{SO}\left(V^{+}\right)$corresponds to the one-dimensional representation of $H^{+}$where $T_{w}$ acts by $q^{l_{0}(w)}$. Thus by Lemma 4, and the isomorphism $H^{+} \cong H_{\psi}^{+}$, the trivial representation of $\mathrm{SO}\left(V^{+}\right)$corresponds to the even Weil representation $\omega_{\psi}^{e}$.

We summarize what we have proven so far:

Corollary 9. In the equivalence of categories $\mathcal{G}_{\psi}^{+}$and $\mathcal{S}_{0}^{+}$, given by the isomorphism of the respective Hecke algebras, square integrable representations are mapped to square integrable representations, and tempered representations are mapped to tempered representations.

\section{HeCKe ALGEBRA $H_{\psi}^{-}$}

Assume that the conductor of $\psi$ is 1 . Consider the $\tilde{K}_{n-1}$ type $\tau_{n-1}$. Recall that this is the Weil representation of $\mathrm{SL}_{2}(\mathfrak{f})$, corresponding to the additive character of $\mathfrak{f}=R / \varpi R$ obtained by restricting $\psi$ to $R$. Let $J=\bigcap_{i=0}^{n-1} K_{i}$, and let $\tilde{J}$ be the inverse image of $J$. In particular, we can view the odd Weil representation $\tau_{n-1}^{o}$ as a representation of $\tilde{J}$ by restriction. Let $H_{\psi}^{-}=H\left(\tau_{n-1}^{o}\right)$ be the Hecke algebra, defined as in Section 7 , for $G=\widetilde{\operatorname{Sp}}(W)_{8}, K=\tilde{J}$ and $\sigma=\tau_{n-1}^{o}$. We shall now determine this algebra in terms of its generator and relations.

First of all, we will show that this algebra is supported on $\tilde{J} W_{a}^{\prime} \tilde{J}$, where $W_{a}^{\prime}$ is the subgroup of $W_{a}$ generated by $s_{0}, \ldots, s_{n-2}$ and $s_{n-1}^{\prime}$, the reflection corresponding to the long root $\alpha_{n-1}^{\prime}=2 e_{n-1}$.

Lemma 10. The algebra $H_{\psi}^{-}$is supported in $\tilde{J} W_{a}^{\prime} \tilde{J}$.

Proof. Recall that $\alpha_{n}=2 e_{n}$. Let $f$ be in $H_{\psi}^{-}$. Let $w \in W_{a}$. Abusing the notation, we consider $w$ as an element in $\widetilde{\operatorname{Sp}}(W)_{8}$. We need to show that if $f(w) \neq 0$ then $w\left(\alpha_{n}\right)= \pm \alpha_{n}$. In particular, either $w \in W_{a}^{\prime}$ or $w s_{n} \in W_{a}^{\prime}$. (Note that $w$ and $w s_{n}$ represent the same $\tilde{J}$-double coset.) Assume that $w\left(\alpha_{n}\right)=\beta+m$ where $m$ is an integer. Let $u \in R$. Then $w^{-1} x_{\alpha_{n}}(u) w=x_{\beta}(t)$ for some $t \in \varpi^{2 m} R$. If $m>0$ or $m=0$ and $\beta$ is a positive root different from $\alpha_{n}$, then $x_{\beta}(t)$ is in $\tilde{J}$ and in the 
kernel of $\tau_{n-1}^{o, *}$. Thus

$$
f(w)=f\left(w x_{\beta}(t)\right)=f\left(x_{\alpha_{n}}(u) w\right)=\tau_{n-1}^{o, *}\left(x_{\alpha_{n}}(u)\right) f(w)
$$

which implies that $\tau_{n-1}^{o, *}\left(x_{\alpha_{n}}(u)\right)$ acts trivially on the image of $f(w)$, for all $u \in R$. Since $\tau_{n-1}^{o, *}$ is cuspidal, this implies that $f(w)=0$. If $m<0$ or $m=0$ and $\beta$ is a negative root different from $-\alpha_{n}$ then we repeat the same argument with $x_{-\alpha_{n}}(u)$ in the place of $x_{\alpha_{n}}(u)$. The lemma is proved.

We now want to prove existence of non-zero elements $T_{i}$ in $H_{\psi}^{-}$, for $i=0, \ldots, n-2$ supported on $\tilde{J} w_{\alpha_{i}}(1) \tilde{J}$ and of a non-zero element $T_{n-1}$ supported on $\tilde{J} w_{\alpha_{n-1}^{\prime}}(1) \tilde{J}$. The case $i \neq n-1$ is easy to treat and similar to what we have done for the algebra $H_{\psi}^{+}$. We normalize these elements so that $T_{i}\left(w_{\alpha_{i}}(1)\right)$, if $i \neq 0$, and $T_{0}\left(w_{\alpha_{0}}^{\prime}(1)\right)$ is the identity in $\operatorname{End}\left(\tau_{n-1}^{o, *}\right)$. The case $i=n-1$ is a real delicacy and we devote our attention to it. Let

$$
K=\bigcap_{i=0}^{n-2} K_{i}
$$

In particular, $J \subseteq K \subseteq K_{n-2}$, and $K$ shares the reductive quotient $\operatorname{Sp}_{4}(\mathfrak{f})$ with $K_{n-2}$. Let $\tilde{K}$ be the inverse image of $K$. Let $H_{\psi, \tilde{K}}$ be the subalgebra of $H_{\psi}^{-}$ consisting of functions supported on $\tilde{K}$. By Lemma 10, this is a one- or twodimensional subalgebra spanned by the identity element of $H_{\psi}^{-}$and $T_{n-1}$, if it exists. The algebra $H_{\psi, \tilde{K}}$ is naturally viewed as the endomorphism algebra of $\operatorname{Ind} \tilde{K}_{\tilde{J}}^{\tilde{K}}\left(\tau_{n-1}^{o, *}\right)$. Thus, in order to show that $T_{n-1}$ exists, it suffices to show that this representation is reducible. Since $\operatorname{Ind}_{\tilde{J}}^{\tilde{K}}\left(\tau_{n-1}^{o, *}\right)$ is dual to $\operatorname{Ind}_{\tilde{J}}^{\tilde{K}}\left(\tau_{n-1}^{o}\right)$ it suffices to show that $\operatorname{Ind}_{\tilde{J}}^{\tilde{K}}\left(\tau_{n-1}^{o}\right)$ is reducible. Since $\tau_{n-1}^{o} \subseteq \tau_{n-2}^{o} \subseteq S\left(X^{*}\right)$, it follows from the Frobenius reciprocity that $\tau_{n-2}^{o}$ is a summand of $\operatorname{Ind}_{\tilde{J}}^{\tilde{K}}\left(\tau_{n-1}^{o}\right)$. This is a proper summand since the dimensions of $\tau_{n-2}^{o}$ and $\operatorname{Ind}_{\tilde{J}}^{\tilde{K}}\left(\tau_{n-1}^{o}\right)$ are $\left(q^{2}-1\right) / 2$ and $\left(q^{4}-1\right) / 2$, respectively. This shows that $T_{n-1}$ exists. Since $w_{\alpha_{n-1}^{\prime}}^{\prime}(1)$ commutes with $x_{ \pm \alpha_{n}}(u)$, $T_{n-1}\left(w_{\alpha_{n-1}^{\prime}}^{\prime}(1)\right)$ must be a scalar in $\operatorname{End}\left(\tau_{n-1}^{o}\right)$. We normalize $T_{n-1}$ so that this scalar is $q^{-\frac{1}{2}}$.

Lemma 11. Let $v_{1}, \ldots, v_{l}$ be a basis of the $\tilde{K}_{n-1}$-type $\tau_{n-1}^{o} \subseteq S\left(X^{*}\right)$, and $v_{1}^{*}, \ldots, v_{l}^{*}$ the dual basis in $\tau_{n-1}^{o, *}$. Then $v=\sum_{i=1}^{l} v_{i} \otimes v_{i}^{*} \in\left(\omega_{\psi}^{o} \otimes \tau_{n-1}^{o, *}\right)^{\tilde{J}}$ and $\omega_{\psi}\left(T_{i}\right) v=q v$ if $i \neq 0$ and $\omega_{\psi}\left(T_{n-1}\right) v=q^{2} v$.

Proof. Let $\langle\cdot, \cdot\rangle$ denote the invariant inner product on $S\left(X^{*}\right)$, as before. Let $\langle\cdot, \cdot\rangle^{*}$ denote an $\tilde{J}$-invariant inner product on $\tau_{n-1}^{o, *}$. In order to simplify expressions, assume that $v_{1}, \ldots, v_{l}$ and $v_{1}^{*}, \ldots, v_{l}^{*}$ are orthonormal bases. Let $(\cdot, \cdot)$ denote the tensor product of the two inner products. As in Lemma 4, the idea is to compute $\left(\omega_{\psi}\left(T_{i}\right) v, v\right)$. Assume first that $i=n-1$. Let $J^{+}$be the pro- $p$ radical of $\tilde{J}$. Since $\tilde{J} w_{\alpha_{n-1}^{\prime}}(1) \tilde{J}=J^{+} w_{\alpha_{n-1}^{\prime}}(1) \tilde{J}$ and $\left[\tilde{J} w_{\alpha_{n-1}^{\prime}}(1) \tilde{J}: \tilde{J}\right]=q^{3}$ there exist elements $x_{j}$ in $J^{+}$such that

$$
\tilde{J} w_{\alpha_{n-1}^{\prime}}(1) \tilde{J}=\bigcup_{j=1}^{q^{3}} x_{j} w_{\alpha_{n-1}^{\prime}}^{\prime}(1) \tilde{J} .
$$


Then, using the definition of $\omega_{\psi}\left(T_{n-1}\right)$,

$$
\omega_{\psi}\left(T_{n-1}\right)\left(v_{i} \otimes v_{i}^{*}\right)=\sum_{j=1}^{q^{3}} x_{j} w_{\alpha_{n-1}^{\prime}}^{\prime}(1) v_{i} \otimes T_{n-1}\left(\left(x_{j} w_{\alpha_{n-1}^{\prime}}^{\prime}(1)\right) v_{i}^{*} .\right.
$$

Since $x_{j} \in J^{+}$and $J^{+}$is in the kernel of $\tau_{n-1}^{o, *}$,

$$
T_{n-1}\left(x_{j} w_{\alpha_{n-1}^{\prime}}^{\prime}(1)\right)=\tau_{n-1}^{o, *}\left(x_{j}\right) \circ T_{n-1}\left(w_{\alpha_{n-1}^{\prime}}^{\prime}(1)\right)=T_{n-1}\left(w_{\alpha_{n-1}^{\prime}}^{\prime}(1)\right) .
$$

Since $T_{n-1}\left(w_{\alpha_{n-1}^{\prime}}^{\prime}(1)\right)$ is the scalar $q^{-\frac{1}{2}}$,

$$
\omega_{\psi}\left(T_{n-1}\right)\left(v_{i} \otimes v_{i}^{*}\right)=q^{-\frac{1}{2}} \sum_{j=1}^{q^{3}} x_{j} w_{\alpha_{n-1}^{\prime}}^{\prime}(1) v_{i} \otimes v_{i}^{*} .
$$

Using orthonormality of the basis $v_{1}^{*}, \ldots, v_{l}^{*}$, it follows that $\left(\omega_{\psi}\left(T_{n-1}\right) v, v\right)$ is equal to

$$
\begin{aligned}
q^{-\frac{1}{2}} \sum_{i=1}^{l} \sum_{j=1}^{q^{3}}\left\langle x_{j} w_{\alpha_{n-1}^{\prime}}^{\prime}(1) v_{i}, v_{i}\right\rangle & =q^{-\frac{1}{2}} \sum_{i=1}^{l} \sum_{j=1}^{q^{3}}\left\langle w_{\alpha_{n-1}^{\prime}}^{\prime}(1) v_{i}, x_{j}^{-1} v_{i}\right\rangle \\
& =q^{\frac{5}{2}} \sum_{i=1}^{l}\left\langle w_{\alpha_{n-1}^{\prime}}^{\prime}(1) v_{i}, v_{i}\right\rangle .
\end{aligned}
$$

We remind the reader that $w_{\alpha_{n-1}^{\prime}}^{\prime}(1)$ is the Fourier transform in the variable $x_{n-1}$. Moreover, since $v_{i}=\varphi^{\prime} \otimes v_{i}^{\prime}$ where $\varphi^{\prime}$ is the characteristic function of the lattice spanned by $\mathbf{e}_{1}^{*}, \ldots, \mathbf{e}_{n-1}^{*}$, it follows that $\left\langle w_{\alpha_{n-1}^{\prime}}^{\prime}(1) v_{i}, v_{i}\right\rangle=q^{-\frac{1}{2}}\left\langle v_{i}, v_{i}\right\rangle$ by Lemma 2 , part (2). Thus $\left(\omega_{\psi}\left(T_{n-1}\right) v, v\right)=q^{2}(v, v)$, as desired. The case $i \neq n-1$ is simpler and left to the reader.

Lemma 12. The operator $T_{i}$ satisfies the quadratic relations $\left(T_{i}-q\right)\left(T_{i}+1\right)=0$ if $i \neq n-1$ and $\left(T_{n-1}-q^{2}\right)\left(T_{n-1}+1\right)=0$. In particular, all $T_{i}$ are invertible.

Proof. We use Lemma 3. The case $i \neq n-1$ is analogous to Lemma 5, so we will omit it. Recall that $T_{n-1}$ is an endomorphism of the induced representation $\operatorname{Ind} \tilde{K}_{\tilde{J}}^{\tilde{K}}\left(\tau_{n-1}^{o, *}\right)$. The dual representation $\operatorname{Ind}_{\tilde{J}}^{\tilde{K}}\left(\tau_{n-1}^{o}\right)$ contains two summands. One of them is $\tau_{n-2}^{o}$. Its dimension is $d_{1}=\left(q^{2}-1\right) / 2$. Thus the other summand has the dimension $d_{2}=q^{2}\left(q^{2}-1\right) / 2$. By Lemma 11, $T_{n-1}$ acts on $\left(\tau_{n-2}^{o} \otimes \tau_{n-1}^{o, *}\right)^{\tilde{J}}$ by $\lambda_{1}=q^{2}$, so it must act on the other summand by $\lambda_{2}=-1$, by Lemma 3, (2). Finally, invertibility of $T_{i}$ follows from the quadratic relation satisfied by $T_{i}$. The lemma is proved.

Theorem 13. Assume that the conductor of $\psi$ is 1 . The Hecke algebra $H_{\psi}^{-}$, as an abstract algebra, is generated by elements $T_{0}, T_{1}, \ldots, T_{n-1}$ satisfying quadratic relations $\left(T_{i}-q\right)\left(T_{i}+1\right)=0$, if $i \neq n-1,\left(T_{n-1}-q^{2}\right)\left(T_{n-1}+1\right)=0$, and satisfying the braid relations corresponding to the following Coxeter diagram:

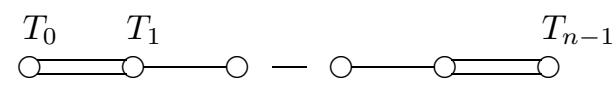


Proof. We need to prove the braid relations. We shall use the weighted length function $l_{3}$ on $W_{a}^{\prime}$. Proposition 1 implies that $J w_{1} J \cdot J w_{2} J=J w_{1} w_{2} J$ if $l_{3}\left(w_{1}\right)+$ $l_{3}\left(w_{2}\right)=l_{3}\left(w_{1} w_{2}\right)$. Since $s_{n-2} s_{n-1}^{\prime} s_{n-2} s_{n-1}^{\prime}=s_{n-1}^{\prime} s_{n-2} s_{n-1}^{\prime} s_{n-2}$ (and both are the shortest expressions in $\left.W_{a}^{\prime}\right)$ it follows that

$$
T_{n-2} T_{n-1} T_{n-2} T_{n-1} \text { and } T_{n-1} T_{n-2} T_{n-1} T_{n-2}
$$

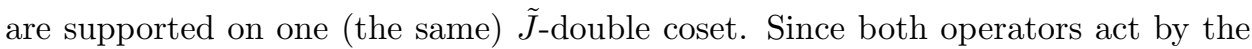
same positive scalar on $\left(\omega_{\psi}^{o} \otimes \tau_{n-1}^{o, *}\right)^{\tilde{J}}$, they have to be equal. This proves one braid relation. Others are proved in the same manner.

If the conductor of $\psi$ is 0 , then the role of $K_{n-1}$ is played by $K_{1}$. We define $J=\bigcap_{i=1}^{n} K_{i}$ and $H_{\psi}^{-}=H\left(\tau_{1}^{o}\right)$ where we view $\tau_{1}^{o}$ as a representation of $\tilde{J}$, by restriction. An obvious analogue of Theorem 13 holds.

\section{Bernstein's COMPOnEnt $\mathcal{G}_{\psi}^{-}$}

Let $P_{o}=M_{o} U_{o}$ be the parabolic subgroup of $\operatorname{Sp}(W)$ defined as the stabilizer of the partial flag $X_{1} \subseteq X_{2} \subseteq \ldots \subseteq X_{n-1}$. Recall that $W_{n-1}$ is the symplectic subspace spanned by $\mathbf{e}_{n}$ and $\mathbf{e}_{n}^{*}$, and $Z_{n-1}^{*}$ is the line spanned by $\mathbf{e}_{n}^{*}$. Let $\omega_{\psi, n-1}$ denote the Weil representation of $\widetilde{\mathrm{Sp}}\left(W_{n-1}\right)_{8}$ acting on $S\left(Z_{n-1}^{*}\right)$. By decomposing $S\left(Z_{n-1}^{*}\right)=S\left(Z_{n-1}^{*}\right)^{e} \oplus S\left(Z_{n-1}^{*}\right)^{o}$ into a sum of spaces of even and odd functions, we have a decomposition $\omega_{\psi, n-1}=\omega_{\psi, n-1}^{e} \oplus \omega_{\psi, n-1}^{o}$ into two irreducible representations. The odd Weil representation $\omega_{\psi, n-1}^{o}$ is supercuspidal.

Let $\widetilde{P}_{o}=\widetilde{M}_{o} U_{o}$ be the inverse image of $P_{o}$ in $\widetilde{\operatorname{Sp}}(W)_{8}$. Any element $m \in \widetilde{M}_{o}$ can be uniquely written as

$$
m=h_{1}^{\prime}\left(t_{1}\right) \cdot \ldots \cdot h_{n-1}^{\prime}\left(t_{n-1}\right) \cdot m^{\prime}
$$

where $m^{\prime}$ is in $\widetilde{\operatorname{Sp}}\left(W_{n-1}\right)_{8}$. Thus, any $n-1$-tuple of characters $\chi=\left(\chi_{1}, \ldots, \chi_{n-1}\right)$ defines a genuine representation $\chi \otimes \omega_{\psi, n-1}^{o}$ of $\widetilde{M}_{o}$.

Let $I\left(\chi \otimes \omega_{\psi, n-1}^{o}\right)$ denote the normalized principal series representation, i.e., we induce the representation $\chi \otimes \omega_{\psi, n-1}^{o}$ multiplied by the modular character $\rho_{U_{o}}^{\frac{1}{2}}$. If $(\pi, V)$ is a smooth representation of $\widetilde{\operatorname{Sp}}(W)_{8}$, let $\left(\pi_{U_{o}}, V_{U_{o}}\right)$ denote the normalized Jacquet module, i.e., the action of $\widetilde{M}_{o}$ on $V_{U_{o}}$ is multiplied by the inverse of the modular character. With these conventions the Frobenius reciprocity takes the form

$$
\operatorname{Hom}_{\widetilde{\mathrm{Sp}}(W)_{8}}\left(V, I\left(\chi \otimes \omega_{\psi, n-1}^{o}\right)\right) \cong \operatorname{Hom}_{\widetilde{M}_{o}}\left(V_{U_{o}}, \chi \otimes \omega_{\psi, n-1}^{o}\right) .
$$

Let $\mathcal{G}_{\psi}^{-}$be the component of the category of smooth representations of $\widetilde{\operatorname{Sp}}(W)_{8}$ whose irreducible representations are submodules of $I\left(\chi \otimes \omega_{\psi, n-1}^{o}\right)$ where $\chi$ is unramified. The odd Weil representation $\omega_{\psi}^{o}$ belongs to $\mathcal{G}_{\psi}^{-}$. Indeed, the map $\ell: S\left(X^{*}\right)^{o} \rightarrow S\left(Z_{n-1}^{*}\right)^{o}$ given by restriction of functions from $X^{*}$ to $Z_{n-1}^{*}$, via the Frobenius reciprocity, gives an embedding of $\omega_{\psi}^{o}$ into $I\left(\chi_{o} \otimes \omega_{\psi, n-1}^{o}\right)$, where $\chi_{o}$ is an unramified character.

Let $\tilde{J}_{M_{o}}=\tilde{J} \cap \widetilde{M}_{o}$, and consider $\tau_{n-1}^{o}$ as a representation of $\tilde{J}_{M_{o}}$ by restriction. By Proposition 3.5.2 in [Be] the natural projection from $V$ to $V_{U_{o}}$ gives a surjection

$$
r:\left(V \otimes \tau_{n-1}^{o, *}\right)^{\tilde{J}} \rightarrow\left(V_{U_{o}} \otimes \tau_{n-1}^{o, *}\right)^{\tilde{J}_{M_{o}}} .
$$

For any $w \in W_{a}^{\prime}$, let $T_{w}=T_{i_{1}} \cdots T_{i_{l}}$ where $w=s_{i_{1}} \cdots s_{i_{l}}$, is a shortest product of simple reflections. Note that $T_{w}$ is invertible since all $T_{i}$ are. Let 
$\lambda=\left(\lambda_{1}, \ldots, \lambda_{n-1}\right) \in \mathbb{Z}^{n-1}$ be a translation in $W_{a}^{\prime}$ such that $\lambda_{1} \geq \ldots \geq \lambda_{n-1} \geq 0$, and let

$$
\hat{T}_{\lambda}=q^{-\frac{l_{2}(\lambda)}{2}} T_{\lambda}
$$

where $l_{2}$ is the weighted length function on $W_{a}^{\prime}$ such that $l_{2}\left(s_{n-1}\right)=2$ and 1 for other simple reflections. Let $t_{\lambda}=h_{1}^{\prime}\left(\varpi^{\lambda_{1}}\right) \cdot \ldots \cdot h_{n-1}^{\prime}\left(\varpi^{\lambda_{n-1}}\right)$. Note that, since $T_{0}\left(w_{\alpha_{n-1}^{\prime}}^{\prime}(1)\right)=q^{-\frac{1}{2}}$, the value of $\hat{T}_{\lambda}$ at $t_{\lambda}$ is $q^{-\frac{l_{3}(\lambda)}{2}}$. Arguing as in Section 9

$$
r \circ \pi\left(\hat{T}_{\lambda}\right)=\pi_{U_{o}}\left(t_{\lambda}\right) \circ r
$$

and, since $T_{\lambda}$ is invertible, the map $r$ is an isomorphism. In particular, if $V$ is an irreducible representation such that $\left(V \otimes \tau_{n-1}^{o, *}\right)^{\tilde{J}} \neq 0$, then $\tau_{n-1}^{o}$ is an $\tilde{J}_{M_{o}}$ type of $V_{U_{o}}$. Since $\tau_{n-1}^{o}$ is the minimal type of $\omega_{\psi, n-1}^{o}$, the Frobenius reciprocity implies that $V$ is a submodule of $I\left(\chi \otimes \omega_{\psi, n-1}^{o}\right)$ for some unramified character $\chi$. Conversely, if $V$ is a submodule of $I\left(\chi \otimes \omega_{\psi, n-1}^{o}\right)$ for some unramified character $\chi$ then $\left(V_{U_{o}} \otimes \tau_{n-1}^{o, *}\right)^{\tilde{J}_{M_{o}}} \neq 0$ and, therefore, $\left(V \otimes \tau_{n-1}^{o, *}\right)^{\tilde{J}} \neq 0$. Thus irreducible representations of $H_{\psi}^{-}$correspond to irreducible constituents of principal series representations $I\left(\chi \otimes \omega_{\psi, n-1}^{o}\right)$. This proves (see (3.12) [BK]):

Corollary 14. The functor that attaches to every smooth representation $V$ of $\widetilde{\mathrm{Sp}}(W)_{8}$ the Hecke algebra module $\left(V \otimes \tau_{n-1}^{o, *}\right)^{\tilde{J}}$ is an equivalence of Bernstein's component $\mathcal{G}_{\psi}^{-}$and the category of $H_{\psi}^{-}$-modules.

Operators $\hat{T}_{\lambda}$ form a commuting family of operators. Thus any finite dimensional representation $F$ of $H_{\psi}^{-}$can be decomposed into a sum of generalized eigenspaces. More precisely, for any $\mu=\left(\mu_{1}, \ldots, \mu_{n-1}\right) \in \mathbb{C}^{n-1}$, define $F_{\mu}$ to be the subspace of $F$ consisting of all $v \in F$ such that

$$
\pi\left(\hat{T}_{\lambda}-q^{-\sum_{i=1}^{n-1} \lambda_{i} \mu_{i}}\right)^{\operatorname{dim}(F)}(v)=0 .
$$

Then $F=\bigoplus F_{\mu}$ and any $\mu$ such that $F_{\mu} \neq 0$ is called an exponent of $F$. The representation $F$ is square integrable if

$$
\Re\left(\mu_{1}\right)>0, \Re\left(\mu_{1}\right)+\Re\left(\mu_{2}\right)>0, \ldots, \Re\left(\mu_{1}\right)+\ldots+\Re\left(\mu_{n-1}\right)>0
$$

for all exponents $\mu$. The representation $F$ is tempered if the exponents satisfy the inequalities where all $>$ have been replaced by $\geq$. These definitions agree with the usual definitions of square integrable and tempered representations of $\widetilde{\operatorname{Sp}}(W)_{8}$.

\section{HeCke Algebra of $\mathrm{SO}\left(V^{-}\right)$}

The affine Hecke algebra of $\mathrm{SO}\left(V^{-}\right)$is described in terms of the affine Weyl group $W_{a}^{\prime}$ (see [Ti]). Recall that $W_{a}^{\prime}$ is generated by by reflections $s_{0}, s_{1}, \ldots, s_{n-1}$ satisfying the braid relations given by the following Coxeter diagram:

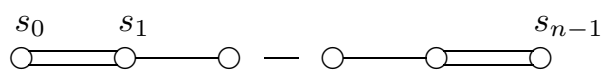

Here the vertex marked $s_{0}$ is special. The quotient of the corresponding special maximal compact subgroup by its pro- $p$ radical is isomorphic to the quasi-split group $\mathrm{O}_{2 n}^{q s}(\mathfrak{f})$. Let $I$ be the Iwahori subgroup of $\mathrm{SO}\left(V^{-}\right)$. It is the pointwise stabilizer of a chamber in the Bruhat-Tits building of $\mathrm{SO}\left(V^{-}\right)$. (In particular $I$ is 
disconnected.) Then $[I w I: I]=q^{l_{2}(w)}$ for every $w$ in $W_{a}^{\prime}$, where $l_{2}$ is the weighted length function on $W_{a}$ such that $l_{2}\left(s_{n-1}\right)=2$ and it is 1 on other simple reflections. Let $H^{-}$denote the Iwahori Hecke algebra of $\mathrm{SO}\left(V^{-}\right)$. For any $w \in W_{a}$ let $T_{w} \in$ $H^{-}$be the characteristic function of the double coset parameterized by $w$. For simplicity, let $T_{i}$ denote $T_{s_{i}}$. Then $T_{i}$ satisfy quadratic relations $\left(T_{i}-q\right)\left(T_{i}+1\right)=0$, if $i \neq n-1,\left(T_{n-1}-q^{2}\right)\left(T_{n-1}+1\right)=0$, and the braid relations given by the above Coxeter diagram. In fact, as an abstract algebra, $H^{-}$is generated by $T_{0}, \ldots, T_{n-1}$ modulo these quadratic and braid relations.

It is worth mentioning that, in contrast with the case of $\mathrm{SO}\left(V^{+}\right)$, the affine Hecke algebra of $\mathrm{SO}\left(V^{-}\right)$is isomorphic to the affine Hecke algebra of its simply connected form. There is also an additional difference worth mentioning. Let $\mathbb{N}: \operatorname{SO}\left(V^{-}\right) \rightarrow k^{\times} /\left(k^{\times}\right)^{2}$ be the spinor norm. Let $\chi_{n r}$ be the unique non-trivial, unramified, quadratic character of $k^{\times}$. Then $\chi_{n r} \circ \mathbb{N}$ and the trivial representation of $\mathrm{SO}\left(V^{-}\right)$belong to two different components of the category of smooth representations of $\mathrm{SO}\left(V^{-}\right)$. Indeed, the restriction of the quadratic character $\chi_{n r} \circ \mathbb{N}$ to the special maximal compact subgroup is the determinant character of the finite group $\mathrm{O}_{2 n}^{q s}(\mathfrak{f})$. Thus, the representation $\chi_{n r} \circ \mathbb{N}$ has no non-zero $I$-fixed vectors. However, each of the two components is equivalent to the category of $\mathrm{H}^{-}$-modules.

Since $H^{-}$and $H_{\psi}^{-}$are given by "identical" combinatorial data (see Theorem[13), we have obtained the following:

Theorem 15. The algebra $H_{\psi}^{-}$is isomorphic, via an explicit isomorphism, to the algebra $H^{-}$.

Let $\lambda=\left(\lambda_{1}, \ldots, \lambda_{n-1}\right) \in \mathbb{Z}^{n-1}$ be a translation in $W_{a}^{\prime}$ such that $\lambda_{1} \geq \ldots \geq$ $\lambda_{n-1} \geq 0$. Define $\hat{T}_{\lambda}=q^{-\frac{l_{2}(\lambda)}{2}} T_{\lambda}$. The operators $\hat{T}_{\lambda}$ form a commuting family of operators and, as we discussed in the previous section, any finite dimensional representation $H^{-}$can be decomposed in a sum of generalized eigenspaces, parameterized by exponents $\mu=\left(\mu_{1}, \ldots, \mu_{n-1}\right) \in \mathbb{C}^{n-1}$. Again, the corresponding representation of $\mathrm{SO}\left(V^{-}\right)$is square integrable if and only if

$$
\Re\left(\mu_{1}\right)>0, \Re\left(\mu_{1}\right)+\Re\left(\mu_{2}\right)>0, \ldots, \Re\left(\mu_{1}\right)+\ldots+\Re\left(\mu_{n-1}\right)>0
$$

for all exponents $\mu$, and it is tempered if the exponents satisfy the inequalities where all $>$ have been replaced by $\geq$.

The trivial representation of $\mathrm{SO}\left(V^{-}\right)$corresponds to the one-dimensional representation of $H^{-}$where $T_{w}$ acts by $q^{l_{2}(w)}$. Thus by Lemma 11, and the isomorphism $H^{-} \cong H_{\psi}^{-}$, the trivial representation of $\mathrm{SO}\left(V^{-}\right)$corresponds to the odd Weil representation $\omega_{\psi}^{o}$.

We summarize what we have proven so far:

Corollary 16. In the equivalence of categories $\mathcal{G}_{\psi}^{-}$and $\mathcal{S}_{0}^{-}$, given by the isomorphism of the respective Hecke algebras, square integrable representations are mapped to square integrable representations.

\section{Steinberg RePRESEntations}

Let $\delta_{\psi}^{\epsilon}$ be a representation of $\widetilde{\mathrm{Sp}}(W)_{8}$ which corresponds to the one-dimensional representation $\delta$ of $H_{\psi}^{\epsilon}$ such that $\delta\left(T_{i}\right)=-1$ for all $i$. Let $v$ be a vector in the unique type in $\delta_{\psi}^{\epsilon}$ isomorphic to $\tau_{0}$ or $\tau_{n-1}^{o}$ if $\epsilon=+$ or $\epsilon=-$, respectively. Let $v^{*}$ be a vector in the dual type in the dual representation. Let $c_{v^{*} v}(g)$ be the corresponding matrix coefficient. Choose $v^{*}$ so that $c_{v^{*} v}(1)=1$. 
Proposition 17. We normalize the Haar measure on $\widetilde{\operatorname{Sp}}(W)_{8}$ so that the measure of $\tilde{I}$ is 1 if $\epsilon=+$ and so that the measure of $\tilde{J}$ is 1 if $\epsilon=-$. Then

(1) Assume that $\epsilon=+$. Let $l_{0}$ be the weighted length function on $W_{a}$ such that $l_{0}\left(s_{0}\right)=0$. Then

$$
\int\left|c_{v^{*} v}(g)\right|^{2} d g=\sum_{w \in W_{a}} \frac{1}{q^{l_{0}(w)}} .
$$

(2) Assume that $\epsilon=-$. Let $l_{2}$ be the weighted length function on $W_{a}^{\prime}$ such that $l_{2}\left(s_{n-1}\right)=2$. Then

$$
\int\left|c_{v^{*} v}(g)\right|^{2} d g=\frac{1}{d\left(\tau_{n-1}^{o}\right)} \sum_{w \in W_{a}^{\prime}} \frac{1}{q^{l_{2}(w)}},
$$

where $d\left(\tau_{n-1}^{o}\right)=(q-1) / 2$ is the dimension of the odd Weil representation $\tau_{n-1}^{o}$ of $\tilde{J}$.

Proof. For (2): Let $J^{+}$be the pro- $p$ radical of $\tilde{J}$. We shall break up the integral over $J^{+}$-cosets. It is easy to see that the matrix coefficient is supported on $\tilde{J} W_{a}^{\prime} \tilde{J}$. Let $g_{1}, \ldots, g_{m}$ be representatives of all $J^{+}$-cosets in $\tilde{J}$. Let $w \in W_{a}^{\prime}$. Then

$$
\tilde{J} w \tilde{J}=\bigcup_{j=1}^{q^{l_{3}(w)}} \bigcup_{i=1}^{m} x_{j} g_{i} w J^{+}
$$

for some $x_{j}$ in $J^{+}$. Then

$$
\begin{aligned}
{\left[\tilde{J}: J^{+}\right] \int\left|c_{v^{*} v}(g)\right|^{2} d g } & =\sum_{w \in W_{a}} \sum_{j=1}^{q^{l_{3}(w)}} \sum_{i=1}^{m}\left|c_{v^{*} v}\left(x_{j} g_{i} w\right)\right|^{2} \\
& =\sum_{w \in W_{a}} q^{l_{3}(w)} \sum_{i=1}^{m}\left|c_{g_{i}^{-1} v^{*} v}(w)\right|^{2} .
\end{aligned}
$$

Claim: $\left|c_{g_{i}^{-1} v^{*} v}(w)\right|^{2}=q^{-l_{2}(w)-l_{3}(w)}\left|c_{g_{i}^{-1} v^{*} v}(1)\right|^{2}$.

Proof of claim: Let $v_{1}, \ldots, v_{l}$ be a basis in $\tau_{n-1}^{o} \subseteq \delta_{\psi}^{-}$. Let $v_{1}^{*}, \ldots, v_{l}^{*}$ be the dual basis in $\tau_{n-1}^{o, *}$. Assume, as we can, that $v=v_{1}$ and $v^{*}=v_{1}^{*}$. Let $w=s_{i_{1}} \cdot \ldots \cdot s_{i_{j}}$ be a shortest expression in terms of simple reflections, and let $T_{w}=T_{s_{i_{1}}} \cdot \ldots \cdot T_{s_{i_{j}}}$. Then

$$
\delta_{\psi}^{-}\left(T_{w}\right)\left(\sum_{i=1}^{l} v_{i} \otimes v_{i}^{*}\right)= \pm \sum_{i=1}^{l} v_{i} \otimes v_{i}^{*} .
$$

On the other hand, pick a representative of $w$ in $\widetilde{\operatorname{Sp}}(W)_{8}$ so that $T_{w}(w)$ is the scalar $q^{\frac{l_{2}(w)-l_{3}(w)}{2}}$. Following the calculation in Lemma 11

$$
\delta_{\psi}^{-}\left(T_{w}\right)\left(\sum_{i=1}^{l} v_{i} \otimes v_{i}^{*}\right)=q^{\frac{l_{2}(w)-l_{3}(w)}{2}} \sum_{i=1}^{l} \sum_{j=1}^{q^{l_{3}(w)}} x_{j} w v_{i} \otimes v_{i}^{*} .
$$

Thus

$$
\pm \sum_{i=1}^{l} v_{i} \otimes v_{i}^{*}=q^{l_{2}(w)-l_{3}(w)} \sum_{i=1}^{l} \sum_{j=1}^{q^{l_{3}(w)}} x_{j} w v_{i} \otimes v_{i}^{*}
$$


Evaluating $g_{i}^{-1} v^{*} \otimes v$ on both sides, using that $x_{j}^{-1}$ fix $g_{i}^{-1} v^{*}$, and squaring both sides proves the claim.

The claim implies that

$$
\int\left|c_{v^{*} v}(g)\right|^{2} d g=\sum_{w \in W_{a}} q^{-l_{2}(w)} \sum_{i=1}^{m} \frac{\left|c_{g_{i}^{-1} v^{*} v}(1)\right|^{2}}{\left[\tilde{J}: J^{+}\right]} .
$$

The inner sum is equal to $1 / d\left(\tau_{n-1}^{o}\right)$ by orthogonality relations of matrix coefficients for the finite group $\tilde{J} / J^{+}$. This proves (2). Part (1) is proved analogously.

Corollary 18. Normalize the Haar measure on $\widetilde{\mathrm{Sp}}(W)_{8}$ so that the measure of $\tilde{I}$ is 1 . Then the formal degrees of $\delta_{\psi}^{+}$and $\delta_{\psi}^{-}$coincide and are equal to the formal degree of the Steinberg representation of $\mathrm{SO}\left(V^{+}\right)$with respect to the Haar measure normalized so that the measure of the Iwahori subgroup is 1 . These numbers are equal to the formal degree of the Steinberg representation of $\mathrm{SO}\left(V^{-}\right)$with respect to the Haar measure normalized so that the measure of the Iwahori subgroup is $2 \cdot \frac{q+1}{q-1}$.

Proof. Let $d$ be the formal degree of the Steinberg representation of $\operatorname{SO}\left(V^{+}\right)$. Then (see $[\mathrm{Bo}]$

$$
\frac{1}{d}=\sum_{w \in W_{a}} \frac{1}{q^{l_{0}(w)}}
$$

and hence $d$ is equal to the formal degree of $\delta_{\psi}^{+}$, by Proposition 17, On the other hand, using Proposition 17 and formulas of Macdonald ([Mc], page 173), one checks that the formal degrees of $\delta_{\psi}^{+}$and $\delta_{\psi}^{-}$are both equal to

$$
\prod_{i=0}^{n-1} \frac{(q-1)\left(q^{n+i}-1\right)}{\left(q^{i+1}-1\right)\left(q^{i+1}+1\right)\left(q^{i}+1\right)} .
$$

Finally, $\sum_{w \in W_{a}^{\prime}} 1 / q^{l_{2}(w)}$ is the inverse of the formal degree of the Steinberg representation of $\mathrm{SO}\left(V^{-}\right)$with respect to the Haar measure normalized so that the measure of the Iwahori subgroup is 1 . Thus, if we change the Haar measure by the factor $2 \cdot \frac{q+1}{q-1}$ then the formal degree is equal to the formal degree of $\delta_{\psi}^{-}$, by Proposition 17.

\section{PARAmeters}

Let $\mathcal{W}_{k}$ be the Weil group of the local field $k$, and let $\mathcal{I}$ be the inertia subgroup of $\mathcal{W}_{k}$. Let Fr denote the Frobenius automorphism. Recall that a Langlands parameter for $\mathrm{SO}\left(V^{+}\right)$(or for $\mathrm{SO}\left(V^{-}\right)$with some restrictions) is a $\mathrm{Sp}_{2 n}(\mathbb{C})$-conjugacy class of homomorphisms

$$
\varphi: \mathcal{W}_{k} \times \mathrm{SL}_{2}(\mathbb{C}) \rightarrow \mathrm{Sp}_{2 n}(\mathbb{C})
$$

such that a finite index subgroup of $\mathcal{I}$ is in the kernel of $\varphi$ and $\varphi\left(\mathcal{W}_{k}\right)$ consists of semi-simple elements. A parameter $\varphi$ is unramified if it is trivial on the inertia subgroup $\mathcal{I}$. It is tempered if $\varphi(\mathrm{Fr})$, when acting on $\mathbb{C}^{2 n}$, has eigenvalues on the unit circle. If $\varphi$ is unramified define a semi-simple element

$$
s=\varphi\left(\operatorname{Fr} \times\left(\begin{array}{cc}
q^{\frac{1}{2}} & 0 \\
0 & q^{-\frac{1}{2}}
\end{array}\right)\right)
$$


and a unipotent element

$$
u=\varphi\left(1 \times\left(\begin{array}{ll}
1 & 1 \\
0 & 1
\end{array}\right)\right) .
$$

These two elements satisfy $\operatorname{sus}^{-1}=u^{q}$, and the conjugacy class of $\varphi$ is determined by the conjugacy class of the pair $(s, u)$. The work of Kazhdan and Lusztig [KL] and of Lusztig $[\mathrm{Lu}$ (Corollary 6.5) gives a parameterization of irreducible representations in $\mathcal{S}_{0}^{+}$and in $\mathcal{S}_{0}^{-}$, respectively, by unramified Langlands parameters.

Proposition 19. The conjugacy class of a tempered, unramified homomorphism $\varphi$ is determined by the conjugacy class of the semi simple element $s$.

Proof. For every non-negative integer $i$, let $V_{i}$ denote the irreducible representation of $\mathrm{SL}_{2}(\mathbb{C})$ of dimension $i+1$. This representation is symplectic if $i$ is odd and orthogonal if $i$ is even. Let $S$ be a finite set of non-negative integers such that, under the action of $\varphi\left(\mathrm{SL}_{2}(\mathbb{C})\right)$,

$$
\mathbb{C}^{2 n}=\bigoplus_{i \in S} W_{i} \otimes V_{i}
$$

where $W_{i}=\operatorname{Hom}_{\mathrm{SL}_{2}(\mathbb{C})}\left(V_{i}, \mathbb{C}^{2 n}\right)$. The restriction of the symplectic form on $\mathbb{C}^{2 n}$ to every isotypic subspace $W_{i} \otimes V_{i}$ is nondegenerate and decomposes as a tensor product of a bilinear form on $W_{i}$ and the unique $\mathrm{SL}_{2}(\mathbb{C})$-invariant form on $V_{i}$. The centralizer of $\varphi\left(\mathrm{SL}_{2}(\mathbb{C})\right)$ in $\operatorname{Sp}_{2 n}(\mathbb{C})$ is the product of classical groups $G\left(W_{i}\right)$. Hence $\varphi(\mathrm{Fr})=\left(s_{i}\right)$ where $s_{i}$ is a semi-simple element in $G\left(W_{i}\right)$. We claim that $s$ determines the decomposition of $\mathbb{C}^{2 n}$ into irreducible representations given above, and the class of $s_{i}$ for all $i$. Indeed, let $j$ be a non-negative integer such that the maximal absolute value of the eigenvalues of $s$ is $q^{j / 2}$. Let $z_{1}, \ldots, z_{m}$ be all eigenvalues of $s$ of absolute value $q^{j / 2}$. Then $j$ is the maximal element in $S$ and $z_{1} q^{-j / 2}, \ldots, z_{m} q^{-j / 2}$ are the eigenvalues of $s_{j}$ on $W_{j}$. We now remove the eigenvalues of $s$ on $W_{j} \otimes V_{j}$ and continue, in the same fashion using the remaining eigenvalues of $s$, to determine the next element in $S$. Since the conjugacy class of $\varphi: \mathrm{SL}_{2}(\mathbb{C}) \rightarrow \mathrm{Sp}_{2 n}(\mathbb{C})$ is completely determined by the decomposition of $\mathbb{C}^{2 n}$ into irreducible representations for $\mathrm{SL}_{2}(\mathbb{C})$, and semi-simple conjugacy classes in the classical groups $G\left(W_{i}\right)$ are determined by their eigenvalues on $W_{i}$, the proposition follows.

We now describe how to attach the parameter $\varphi$ to a representation $\pi$ in these two components in three steps:

(1) Attach $s$ to $\pi$.

(2) Attach $\varphi$ to $\pi$ if $\pi$ is tempered.

(3) Attach $\varphi$ to $\pi$ if $\pi$ is a Langlands quotient.

(1) Assume first that $\pi$ is an irreducible representation in $\mathcal{S}_{0}^{+}$. Recall, from Section 2, the maximal split torus $T_{n}=\left(k^{*}\right)^{n}$ of $\operatorname{SO}\left(V^{+}\right)$. Let $\chi=\left(\chi_{1}, \ldots, \chi_{n}\right)$ be an $n$-tuple of unramified characters. It defines an unramified character $\chi$ of $T_{n}$ by $\chi(t)=\chi_{1}\left(t_{1}\right) \cdots \chi_{n}\left(t_{n}\right)$ for every $t=\left(t_{1}, \ldots, t_{n}\right) \in T_{n}$. If $\pi$ is a subquotient of the principal series obtained by inducing $\chi$ then $s$, acting on the standard representation of $\operatorname{Sp}_{2 n}(\mathbb{C})$, has eigenvalues $\chi_{1}(\varpi)^{ \pm 1}, \ldots, \chi_{n}(\varpi)^{ \pm 1}$. Now assume that $\pi$ is an irreducible representation in $\mathcal{S}_{0}^{-}$. Recall, from Section 2 the maximal split torus $T_{n-1}=\left(k^{*}\right)^{n-1}$ of $\mathrm{SO}\left(V^{-}\right)$. Its centralizer is $T_{n-1} \times\left(D^{*} / k^{*}\right)$ where $D$ is a quaternion algebra over $k$. This is a Levi factor of a minimal parabolic subgroup of $\mathrm{SO}\left(V^{-}\right)$. An unramified character of this Levi factor is, by definition, trivial on 
the compact factor $\left(D^{*} / k^{*}\right)$ and is thus given by an $n-1$-tuple $\chi=\left(\chi_{1}, \ldots, \chi_{n-1}\right)$ of unramified characters. In this case $s$ has eigenvalues $\chi_{1}(\varpi)^{ \pm 1}, \ldots, \chi_{n-1}(\varpi)^{ \pm 1}$ and $q^{ \pm \frac{1}{2}}$.

(2) If $\pi$ is tempered then Proposition 19 shows how $\varphi$ is determined by $s$.

(3) A general representation of $\mathrm{SO}(V)$ ( $V$ denotes $V^{+}$or $V^{-}$here) can be described as a Langlands quotient as follows. As in Section 2, let $Q$ be the maximal parabolic subgroup of $\mathrm{SO}(V)$ defined as the stabilizer of the $l$-dimensional totally isotropic subspace $Y_{l}$, with the Levi factor $L=\mathrm{GL}\left(Y_{l}\right) \times \mathrm{SO}\left(V_{l}\right)$. Let $\sigma$ be a Langlands quotient for $\mathrm{GL}\left(Y_{l}\right)$ obtained by inducing $\tau_{1}^{s_{1}} \otimes \cdots \otimes \tau_{r}^{s_{r}}$ from a parabolic subgroup stabilizing a partial flag

$$
Y_{l_{1}} \subseteq Y_{l_{2}} \subseteq \ldots \subseteq Y_{l_{r}}=Y_{l}
$$

where $\tau_{i}^{s_{i}}$ is a twist by $\left|\operatorname{det}_{Y_{l_{i}} / Y_{l_{i-1}}}\right|^{s_{i}}$ of a unitary discrete series representation $\tau_{i}$ of $\operatorname{GL}\left(Y_{l_{i}} / Y_{l_{i-1}}\right)$, and $s_{i}$ are complex numbers satisfying $\Re\left(s_{1}\right) \geq \Re\left(s_{2}\right) \ldots \geq \Re\left(s_{r}\right)>$ 0 . Let $\tau$ be an irreducible tempered representation of $\mathrm{SO}\left(V_{l}\right)$. Let $I_{Q}(\sigma \otimes \tau)$ be the representation of $\mathrm{SO}(V)$ obtained by inducing $\sigma \otimes \tau$ from $Q$. The representation $I_{Q}(\sigma \otimes \tau)$ has a unique irreducible quotient denoted by $J_{Q}(\sigma \otimes \tau)$. Every irreducible representation $\pi$ is isomorphic to $J_{Q}(\sigma \otimes \tau)$ for some triple $(Q, \sigma, \tau)$. If $\pi$ has a non-zero Iwahori-fixed vector, then $\tau_{i}$ is a Steinberg representation for every $i$, and $\tau$ has a non-zero Iwahori-fixed vector. The Langlands parameter of $\pi$ is

$$
\varphi=\varphi_{\sigma}+\varphi_{\tau}+\varphi_{\sigma}^{*}
$$

where $\varphi_{\tau}$ is the Langlands parameter of $\tau$ and $\varphi_{\sigma}$ is the Langlands parameter of $\sigma$.

By our equivalence of categories, we obtain a parameterization of irreducible representations in $\mathcal{G}_{\psi}^{+}$and $\mathcal{G}_{\psi}^{-}$. Since we shall need it in the next section, we shall now sketch how to attach unramified parameters to representations in these two components. Again, the semi-simple class $s$ is easy to describe. If $\pi$ is an irreducible representation in $\mathcal{G}_{\psi}^{+}$, then $\pi$ is a subquotient of an induced representation $I(\chi)$ where $\chi=\left(\chi_{1}, \ldots, \chi_{n}\right)$ is an $n$-tuple of unramified characters. Then $s$ has eigenvalues $\chi_{1}(\varpi)^{ \pm 1}, \ldots, \chi_{n}(\varpi)^{ \pm 1}$. If $\pi$ is an irreducible representation in $\mathcal{G}_{\psi}^{-}$, then $\pi$ is a subquotient of an induced representation $I\left(\chi \otimes \omega_{\psi, n-1}^{o}\right)$ where $\chi=\left(\chi_{1}, \ldots, \chi_{n-1}\right)$ is an $n-1$-tuple of unramified characters. In this case $s$ has eigenvalues $\chi_{1}(\varpi)^{ \pm 1}, \ldots, \chi_{n-1}(\varpi)^{ \pm 1}$ and $q^{ \pm \frac{1}{2}}$. If $\pi$ is tempered, then $s$ determines $u$, just as in the case of $\mathrm{SO}(V)$. For all other representations we need a description in terms of Langlands quotients.

As in Section 2, let $P$ be the maximal parabolic subgroup of $\operatorname{Sp}(W)$ defined as the stabilizer of the $l$-dimensional totally isotropic subspace $X_{l}$. Let $\widetilde{P}=\widetilde{M} U_{P}$ be the inverse image of $P$ in $\widetilde{\mathrm{Sp}}(W)_{8}$ where $\widetilde{M}=\mathrm{GL}\left(X_{l}\right) \times \widetilde{\mathrm{Sp}}\left(W_{l}\right)_{8}$ is the Levi factor. Let $\sigma$ be a Langlands quotient for $\operatorname{GL}\left(X_{l}\right)$, as above, and let $\tau$ be an irreducible tempered representation of $\widetilde{\mathrm{Sp}}\left(W_{l}\right)_{8}$. Let $I_{P}(\sigma \otimes \tau)$ be the representation of $\widetilde{\mathrm{Sp}}(W)_{8}$ obtained by inducing $\sigma \otimes \tau$ from $\widetilde{P}$. The representation $I_{P}(\sigma \otimes \tau)$ has a unique irreducible quotient denoted by $J_{P}(\sigma \otimes \tau)$. Every irreducible representation $\pi$ is isomorphic to $J_{P}(\sigma \otimes \tau)$ for some triple $(P, \sigma, \tau)$. If $\pi$ is in $\mathcal{G}_{\psi}^{+}$then $\tau$ is in the Bernstein component of $\widetilde{\operatorname{Sp}}\left(W_{l}\right)_{8}$ that contains $\omega_{\psi, l}^{e} \cong\left(\omega_{\psi}^{e}\right)_{U_{P}}$. Similarly, if $\pi$ is in $\mathcal{G}_{\psi}^{-}$then $\tau$ is in the Bernstein component of $\widetilde{\operatorname{Sp}}\left(W_{l}\right)_{8}$ that contains $\omega_{\psi, l}^{o} \cong\left(\omega_{\psi}^{o}\right)_{U_{P}}$. (In particular $l<n$.) In any case, the Langlands parameter of $\pi$ is $\varphi=\varphi_{\sigma}+\varphi_{\tau}+\varphi_{\sigma}^{*}$. 


\section{Theta correspondence}

The goal of this section is to show that the theta correspondence preserves unramified Langlands parameters. Let $(V, Q)$ be an orthogonal space of dimension $2 n+1$. Then $(\operatorname{Sp}(W), \mathrm{O}(V))$ is a dual pair in $\operatorname{Sp}(W \otimes V)$. Note that $X^{*} \otimes V$ is a maximal totally isotropic subspace of $W \otimes V$. Let $S\left(X^{*} \otimes V\right)$ be the space of locally constant, compactly supported functions on $X^{*} \otimes V$. The Weil representation, defined by means of the additive character $\psi$, is a representation of $\operatorname{Sp}(W \otimes V)^{\prime \prime} \subseteq \operatorname{GL}\left(S\left(X^{*} \otimes V\right)\right)$, a central extension of $\operatorname{Sp}(W \otimes V)$ by $\mathbb{C}^{\times}$. We consider $\mathrm{O}(V)$ as a subgroup of $\mathrm{Sp}(W \otimes V)^{\prime \prime}$ so that for any $g \in \mathrm{O}(V)$ and $f \in S\left(X^{*} \otimes V\right)$

$$
[g \cdot f](x \otimes v)=f\left(x \otimes g^{-1} v\right) .
$$

Let $\operatorname{Sp}(W)^{\prime \prime} \subseteq \operatorname{Sp}(W \otimes V)^{\prime \prime}$ be the inverse image of $\operatorname{Sp}(W)$. Since $\operatorname{Sp}(W)$ is perfect, the inclusion of $\operatorname{Sp}(W)$ into $\operatorname{Sp}(W \otimes V)$ lifts uniquely to an isomorphism $i$ of $\operatorname{Sp}(W)^{\prime}$ and $\operatorname{Sp}(W)^{\prime \prime}$ such that $i(\zeta)=\zeta$ for all $\zeta \in \mathbb{C}^{\times}$. Obviously, the map $i$ is determined by its values on $x_{\alpha}(u)$ and $x_{-\alpha}(u)$ for all simple roots $\alpha$. If $\alpha$ is short, then these elements generate a subgroup of $\operatorname{Sp}(W)^{\prime}$ isomorphic to $\mathrm{SL}_{2}(k)$. For any $g$ in this group $i(g)$ is necessarily given by

$$
[i(g) \cdot f](x \otimes v)=f\left(g^{-1} x \otimes v\right) .
$$

Now assume that $\alpha$ is a long root. For ease of notation assume that $\operatorname{dim}(W)=2$. Then $\operatorname{Sp}^{\prime \prime}(W)$ contains operators defined by

and

$$
\begin{gathered}
{\left[x_{\alpha}^{\prime \prime}(u) f\right](v)=\psi(u Q(v)) f(v),} \\
{\left[h_{\alpha}^{\prime \prime}(t) f\right](v)=|t|^{\frac{2 n+1}{2}} f(t v),}
\end{gathered}
$$

$$
\left[w_{\alpha}^{\prime \prime}(-1 / t) f\right](v)=\left[w_{-\alpha}^{\prime \prime}(t) f\right](v)=|t|^{\frac{2 n+1}{2}} \int_{k} f(t y) \psi(\langle v, y\rangle) d y
$$

where $f \in S(V)$ and $\langle\cdot, \cdot\rangle$ is a symmetric bilinear form such that $2 Q(v)=\langle v, v\rangle$. These elements differ from $i\left(x_{\alpha}(u)\right), i\left(h_{\alpha}(t)\right)$ and $i\left(w_{\alpha}(t)\right)$ by non-zero complex numbers. As in Section 5 one argues that $i\left(x_{\alpha}(u)\right)=x_{\alpha}^{\prime \prime}(u)$, and there exists an eight root of 1 , the Weil index $\gamma\left(\psi_{t} \circ Q\right)$, such that

$$
i\left(w_{\alpha}(t)\right)=\gamma\left(\psi_{t} \circ Q\right) \cdot w_{\alpha}^{\prime \prime}(t) .
$$

It follows that $i\left(h_{\alpha}(t)\right)=\gamma(t, \psi \circ Q) h_{\alpha}^{\prime \prime}(t)$, where $\gamma(t, \psi \circ Q)=\gamma\left(\psi_{t} \circ Q\right) / \gamma(\psi \circ Q)$.

Now assume that $(-1)^{n} \operatorname{det}(V)$, the discriminant of $V$, is 1 . Then Lemma 4.2, Chapter I, in Kudla's Hirschberg Castle notes $\mathrm{Ku2}$ shows that $\gamma(t, \psi \circ Q)=\gamma(t, \psi \circ$ $\left.x^{2}\right)$. Thus $i\left(h^{\prime}(t)\right)=h^{\prime \prime}(t)$ where $h^{\prime}(t)$ is defined in Section 5 .

In particular, if the discriminant of $V$ is 1 , then $-1_{V} \in \mathrm{O}(V)$ and $-1_{W}=$ $h_{1}^{\prime}(-1) \cdots h_{n}^{\prime}(-1) \in \operatorname{Sp}(W)^{\prime}$ coincide as elements in $\operatorname{Sp}(W \otimes V)^{\prime \prime}$. Note that $-1_{W}$ can be defined canonically as the unique element in $\operatorname{Sp}(W)^{\prime}$ acting as 1 on $\omega_{\psi}^{e}$ and as -1 on $\omega_{\psi}^{o}$. In the rest of this section we assume that the discriminant of $V$ is 1 .

Let $\pi$ be an irreducible representation of $\mathrm{SO}(V)$. Since $\mathrm{O}(V) \cong\left\{ \pm 1_{V}\right\} \times \mathrm{SO}(V)$, $\pi$ can be extended to $\mathrm{O}(V)$ in two different ways. Let $\epsilon(V)$ be the Hasse invariant of $V$, i.e., $\epsilon\left(V^{+}\right)=1$ and $\epsilon\left(V^{-}\right)=-1$. Let $\epsilon(s, \pi, \psi)$ be the standard epsilon factor attached to $\pi$. Its value at $1 / 2$ does not depend on $\psi$, and it is equal to 1 or -1 . We extend $\pi$ to $\mathrm{O}(V)$ so that $-1_{V}$ acts as $\epsilon(V) \epsilon(1 / 2, \pi, \psi)$. This and only this extension has a non-zero, irreducible theta lift to $\operatorname{Sp}(W)^{\prime}$ denoted by $\theta_{W, V}(\pi)$ (see [GS]). It follows that $-1_{W}$ acts as $\epsilon(V) \epsilon(1 / 2, \pi, \psi)$ on $\theta_{W, V}(\pi)$. In the notation of Section 2 and Section 15, the following is proved in [GI and GS]: 
Theorem 20. Let $\pi$ be an irreducible representation of $\mathrm{SO}(V)$.

(1) If $\pi$ is tempered then $\theta_{W, V}(\pi)$ is tempered.

(2) If $\pi \cong J_{Q}(\sigma \otimes \tau)$ then $\theta_{W, V}(\pi) \cong J_{P}\left(\sigma \otimes \theta_{W_{l}, V_{l}}(\tau)\right)$.

We now have:

Corollary 21. Let $\pi$ be an irreducible representation of $\mathrm{SO}(V)$ containing non-zero Iwahori-fixed vectors. Then $\theta_{W, V}(\pi)$ is in $\mathcal{G}_{\psi}^{+}$or in $\mathcal{G}_{\psi}^{-}$and the Langlands parameter of $\theta_{W, V}(\pi)$, as defined in Section 15, coincides with the Langlands parameter of $\pi$. In particular, the theta correspondence gives a bijection of finite sets $\operatorname{Irr}(s, u)$ and $\operatorname{Irr}_{\psi}(s, u)$ for all pairs $(s, u)$.

Proof. Kudla's Ku1 results on the supercuspidal support for theta correspondences imply that $\theta_{W, V}(\pi)$ must be in one of the two components, and the semi-simple class $s$ is preserved. If $\pi$ is tempered then $\theta_{W, V}(\pi)$ is also tempered, by Theorem 20. (1). In this case the unipotent class is uniquely determined by $s$ (see Section [15), so the parameters of $\pi$ and $\theta_{W, V}(\pi)$ are the same. The general case follows from Theorem 20, (2).

Although the theta correspondence preservers the parameter $(s, u)$, it does not coincide with the equivalence of categories. This can be seen on the following important example. Assume that the pair $(s, u)$ corresponds to the unique parameter $\varphi$ trivial on $W_{k}$ and such that $u$ belongs to the regular unipotent orbit. Let $\mathrm{St}^{ \pm}$be the Steinberg representation of $\mathrm{SO}\left(V^{ \pm}\right)$. Then

$$
\operatorname{Irr}(s, u)=\left\{\mathrm{St}^{+}, \mathrm{St}^{-}\right\} \quad \text { and } \operatorname{Irr}_{\psi}(s, u)=\left\{\delta_{\psi}^{+}, \delta_{\psi}^{-}\right\}
$$

where $\delta_{\psi}^{ \pm}$are the square integrable representations studied in Section 14, Note that $-1_{W}$ acts as 1 on $\omega_{\psi}^{e}$ and as -1 on $\omega_{\psi}^{o}$. It follows that $-1_{W}$ acts as 1 on any representation in $\mathcal{G}_{\psi}^{+}$and as -1 on any representation in $\mathcal{G}_{\psi}^{-}$. In particular, $-1_{W}$ acts as -1 on $\delta_{\psi}^{-}$. On the other hand, since $\epsilon\left(V^{+}\right)=1$ and $\epsilon\left(1 / 2, \mathrm{St}^{+}, \psi\right)=-1$, it follows that $-1_{W}$ acts as -1 on $\theta_{W, V^{+}}\left(\mathrm{St}^{+}\right)$. This forces

$$
\theta_{W, V^{+}}\left(\mathrm{St}^{+}\right) \cong \delta_{\psi}^{-} \text {and } \theta_{W, V^{-}}\left(\mathrm{St}^{-}\right) \cong \delta_{\psi}^{+} \text {. }
$$

Now let $\chi$ be a non-trivial unramified quadratic character of $k^{*}$. Using the Hilbert symbol we can write $\chi(x)=(x, a)$ for some non-square unit $a$. Let $\mathrm{St}_{\chi}^{ \pm}$be the quadratic twist of $\mathrm{St}^{ \pm}$by $\chi$. Let $\delta_{\psi_{a}}^{ \pm}$be the square integrable representation of $\operatorname{Sp}(W)^{\prime}$ which corresponds to $\mathrm{St}^{ \pm}$via the equivalence of components $\mathcal{G}_{\psi_{a}}^{ \pm}$and $\mathcal{S}_{0}^{ \pm}$. Since $a$ is a unit, the components $\mathcal{G}_{\psi}^{+}$and $\mathcal{G}_{\psi_{a}}^{+}$coincide, and $\delta_{\psi_{a}}^{+}$corresponds to $\mathrm{St}_{\chi}^{+}$ by the equivalence of $\mathcal{G}_{\psi}^{+}$and $\mathcal{S}_{0}^{+}$. On the other hand, $\delta_{\psi_{a}}^{-}$belongs to the component $\mathcal{G}_{\psi_{a}}^{-}$which is different but equivalent to $\mathcal{G}_{\psi}^{-}$, just as $\mathrm{St}_{\chi}^{-}$belongs to a component of $\mathrm{SO}\left(V^{-}\right)$which is different but equivalent to $\mathcal{S}_{0}^{-}$. Since $\epsilon\left(1 / 2, \mathrm{St}_{\chi}^{+}, \psi\right)=1$, it is not difficult to show that

$$
\theta_{W, V^{+}}\left(\mathrm{St}_{\chi}^{+}\right) \cong \delta_{\psi_{a}}^{+} \text {and } \theta_{W, V^{-}}\left(\mathrm{St}_{\chi}^{-}\right) \cong \delta_{\psi_{a}}^{-}
$$

\section{REFERENCES}

[Au] A. M. Aubert, Description de la correspondance de Howe en termes de classification de Kazhdan-Lusztig. Invent. Math. 103 (1991) 379-415. MR1085113 (92h:22036)

[Be] J. N. Bernstein (rédigé par P. Deligne), Le "centre" de Bernstein. Représentations des groupes réductif sur un corps local (Hermann, Paris, 1984) 1-32. MR771671 (86e:22028) 
[Bo] A. Borel, Admissible representations of a semi-simple group over a local field with vectors fixed under an Iwahori subgroup. Invent. Math. 35, (1976) 233-259. MR0444849 (56:3196)

[BK] C. J. Bushnell and P. C. Kutzko, Smooth representations of reductive p-adic groups: structure theory via types. Proc. London Math. Soc. (3) 77 (1998) 582-634. MR1643417 (2000c:22014)

[GI] W. T. Gan and A. Ichino, Formal degrees and local theta correspondences. Preprint.

[GS] W. T. Gan and G. Savin, Representations of metaplectic groups I: epsilon dichotomy and local Langlands correspondence. To appear in Comp. Math.

[IM] N. Iwahori and H. Matsumoto, On some Bruhat decomposition and the structure of the Hecke rings of p-adic Chevalley groups. Pub. Math. IHES, 25 (1965) 5-48. MR0185016 (32:2486)

[KL] D. Kazhdan and G. Lusztig, Proof of the Deligne-Langlands conjecture for Hecke algebras. Invent. Math. 87 (1987) 153-215. MR862716 (88d:11121)

[Ku1] S. Kudla, On the local theta correspondence. Invent. Math. 83 (1986) 229-255. MR818351 (87e:22037)

[Ku2] S. Kudla, Notes on the local theta correspondence. Lecture Notes from the European School of Group Theory, available at http://www.math.toronto.edu/ skudla/ssk.research.html

[Lu] G. Lusztig, Unipotent representations of simple p-adic groups. Inter. Math. Res. Not. 11 (1995) 517-589. MR1369407 (98b:22034)

[Mc] I. G. Macdonald, The Poincaré Series of a Coxeter Group. Math. Ann. 199 (1972) 161-174. MR0322069 (48:433)

[Ma] H. Matsumoto, Sur les sous-groupes arithmétiques des groupes semi-simples déployés. Ann. Sci. École Norm. Sup. Sér. 4, 2 no. 1 (1969) 1-62 . MR0240214 (39:1566)

[Mo] C. C. Moore, Group extensions of $p$-adic and adelic linear groups. Pub. Math. IHES, 35 (1968) 5-70. MR0244258 (39:5575)

[Ro] J. Rogawski, On modules over the Hecke algebra of a p-adic group. Invent. Math. 79 (1985) 443-465. MR782228 (86j:22028)

[Sa] G. Savin, On unramified representations of covering groups. J. Reine and Angew. Math. 566 (2004) 111-134. MR2039325 (2005a:22014)

[St] R. Steinberg, Lectures on Chevalley groups. Yale University (1968). MR0466335 (57:6215)

[Ti] J. Tits, Reductive groups over local fields. Automorphic forms, representations and $L$ functions (Proc. Sympos. Pure Math., Oregon State Univ., Corvallis Ore., 1977), Part 1, pp 29-69, Proc. Sympos. Pure Math., XXXIII, AMS, Providence, R.I., 1979. MR546588 (80h:20064)

[We] A. Weil, Sur certains groupes d'opérateurs unitaires, Acta Math. 111 (1964) 143-211. MR0165033 (29:2324)

Department of Mathematics, University of California at San Diego, 9500 Gilman Drive, La Jolla, California 92093 - and - Department of Mathematics, National University of Singapore, 10 Lower Kent Ridge Road, Singapore 119076

E-mail address: wgan@math.ucsd.edu

Department of Mathematics, University of Utah, 155 South 1400 East, Salt Lake City, UTAH 84112

E-mail address: savin@math.utah.edu 